\section{European Myeloma Network review and consensus statement on smoldering multiple myeloma: how to distinguish (and manage) Dr. Jekyll and Mr. Hyde}

\author{
Pellegrino Musto, ${ }^{1}$ Monika Engelhardt, ${ }^{2}$ Jo Caers, ${ }^{3,4}$ Niccolo' Bolli, $, 5,6$ Martin \\ Kaiser, ${ }^{7,8}$ Niels van de Donk, ${ }^{9}$ Evangelos Terpos, ${ }^{10}$ Annemiek Broijl, ${ }^{11}$ Carlos \\ Fernández de Larrea, ${ }^{12}$ Francesca Gay, ${ }^{13}$ Hartmut Goldschmidt, ${ }^{14}$ Roman \\ Hajek, ${ }^{15}$ Annette Juul Vangsted, ${ }^{16}$ Elena Zamagni, ${ }^{17}$ Sonja Zweegman, ${ }^{9}$ Michele \\ Cavo ${ }^{17}$ Meletios Dimopoulos ${ }^{18}$ Hermann Einsele, ${ }^{19}$ Heinz Ludwig, ${ }^{20}$ Giovanni \\ Barosi, ${ }^{21}$ Mario Boccadoro, ${ }^{13}$ Maria-Victoria Mateos, ${ }^{22}$ Pieter Sonneveld ${ }^{11}$ and \\ Jesus San Miguel ${ }^{23}$
}

1"Aldo Moro" University School of Medicine, Unit of Hematology and Stem Cell Transplantation, AOUC Policlinico, Bari, Italy; ${ }^{2}$ Department of Medicine I, Medical Center - University of Freiburg, Freiburg, Faculty of Medicine, University of Freiburg, Germany; ${ }^{3}$ Department of Clinical Hematology, CHU of Liège, Liège, Belgium; ${ }^{4}$ Laboratory of Hematology, GIGA-I3, University of Liège, Liège, Belgium; ${ }^{5}$ Division of Hematology, Fondazione IRCCS Ca' Granda Ospedale Maggiore Policlinico, Milano, Italy; ${ }^{6}$ Department of Oncology and Onco-Hematology, University of Milan, Milano, Italy; ${ }^{7}$ The Institute of Cancer Research, Division of Molecular Pathology, London, UK; ${ }^{8}$ The Royal Marsden Hospital, Department of Haematology, London, UK; ${ }^{9}$ Amsterdam UMC, Vrije Universiteit Amsterdam, Department of Hematology, Cancer Center Amsterdam, Amsterdam, the Netherlands; ${ }^{10}$ Stem Cell Transplantation Unit, Plasma Cell Dyscrasias Unit, Department of Clinical Therapeutics, School of Medicine, National and Kapodistrian University of Athens, Alexandra General Hospital, Athens, Greece; ${ }^{11}$ Erasmus MC Cancer Institute \& Erasmus University of Rotterdam, Rotterdam, the Netherlands; ${ }^{12}$ Amyloidosis and Myeloma Unit, Department of Hematology, Hospital Clínic, IDIBAPS, University of Barcelona, Barcelona, Spain; ${ }^{13}$ Myeloma Unit, Division of Hematology, University of Torino, Azienda Ospedaliero Universitaria Città della Salute e della Scienza, Torino, Italy; ${ }^{14}$ University Hospital Heidelberg Internal Medicine V and National Center for Tumor Diseases (NCT), Heidelberg, Germany; ${ }^{15}$ Department of Hemato-oncology, University Hospital Ostrava and Faculty of Medicine, University of Ostrava, Ostrava, Czech Republic; ${ }^{16}$ Department of Hematology, Rigshospitalet, Copenhagen, Denmark; ${ }^{17}$ Seràgnoli Institute of Hematology, Bologna University School of Medicine, Bologna, Italy; ${ }^{18} \mathrm{National}$ and Kapodistrian University of Athens, School of Medicine, Department of Clinical Therapeutics, Athens, Greece; ${ }^{19}$ University Hospital Würzburg, Internal Medicine II, Würzburg, Germany; ${ }^{20}$ Wilhelminen Cancer Research Institute, 1st Department of Medicine, Center for Oncology, Hematology and Palliative Care, Wilhelminenspital, Vienna, Austria; ${ }^{21}$ Fondazione IRCCS Policlinico S. Matteo, Pavia, Italy; ${ }^{22}$ University Hospital of Salamanca/IBSAL/CIC-IBMCC, Salamanca, Spain and ${ }^{23}$ Universidad de Navarra, CIMA, IDISNA, CIBERONC, Pamplona, Spain

\section{ABSTRACT}

A ccording to the updated International Myeloma Working Group criteria, smoldering multiple myeloma (SMM) is an asymptomatic plasma cell disorder characterized by an M-component $>3 \mathrm{~g} / \mathrm{dL}$, bone marrow plasma cell infiltration $>10 \%$ and $<60 \%$, and absence of any myeloma-defining event. Active multiple myeloma is preceded by SMM, with a median time to progression of approximately 5 years. Cases of SMM range from the extremes of "monoclonal gammopathy of undetermined significance-like", in which patients never progress during their lifetimes, to "early multiple myeloma", in which transformation into symptomatic disease, based on genomic evolution, may be rapid and devastating. Such a "split personality" makes the prognosis and management of individual patients challenging, particularly with regard to the identification and possible early treatment of high-risk SMM. Outside of clinical trials, the conventional approach to SMM generally remains close observation until progression to active multiple myeloma. However, two prospective, randomized trials have recently demonstrated a significant clinical benefit in terms of time to progression, and of
Ferrata Storti Foundation

Haematologica 2021

Volume 106(11):2799-2812

Correspondence:

PELLEGRINO MUSTO

p.musto@tin.it

Received: March 12, 2021.

Accepted: June 15, 2021

Pre-published: July 15, 2021.

https://doi.org/10.3324/haematol.2021.278519

(C)2021 Ferrata Storti Foundation

Material published in Haematologica is covered by copyright. All rights are reserved to the Ferrata Storti Foundation. Use of published material is allowed under the following terms and conditions:

https://creativecommons.org/licenses/by-nc/4.0/legalcode. Copies of published material are allowed for personal or internal use. Sharing published material for non-commercial purposes is subject to the following conditions:

https://creativecommons.org/licenses/by-nc/4.0/legalcode, sect. 3. Reproducing and sharing published material for commercial purposes is not allowed without permission in writing from the publisher. 
overall survival in one of the two studies, for some patients with higher-risk SMM treated with lenalidomide \pm dexamethasone, raising the question of whether such an approach should be considered a new standard of care. In this paper, experts from the European Myeloma Network describe current biological and clinical knowledge on SMM, focusing on novel insights into its molecular pathogenesis, new prognostic scoring systems proposed to identify SMM patients at higher risk of early transformation, and updated results of completed or ongoing clinical trials. Finally, some practical recommendations for the real-life management of these patients, based on Delphi consensus methodology, are provided.

\section{Introduction}

"Asymptomatic", smoldering multiple myeloma (SMM) is in the middle of the continuum of monoclonal gammopathies. SMM is more advanced and carries a higher disease burden than monoclonal gammopathy of undetermined significance (MGUS), but does not show the clinical features of end-organ damage, nor any of the other myeloma-defining events of active multiple myeloma (MM). $\cdot^{1.5}$

Of all patients with MM, 8-14\% have SMM: these patients have a median age of onset of 67 years, an annual incidence of 0.4 cases per 100,000 persons and a higher prevalence in Americans of African descent. ${ }^{6,7}$ The median time to progression to active $\mathrm{MM}$ is around 5 years, with a variable rate of approximately $10 \%$ /year during the first 5 years, 3\%/year for the next 5 years, and $1 \%$ /year thereafter. ${ }^{7.8}$ Thus, SMM is a heterogeneous entity, ranging from 'MGUS-like' to 'early MM', in which malignant plasma cells can rapidly expand and lead to active MM. Yet, nearly one third of cases of SMM will never progress. Such a "split personality" of SMM remains intriguing and challenging.

For decades, the conventional approach to SMM has been close observation, delaying treatment to the time of progression to active MM. Significant advances in the understanding of disease biology, improved risk stratification, and newer therapies with better efficacy and lower toxicity contributed to deeper responses and longer survival for patients with active MM. These advances have also challenged the management of SMM, raising the question of whether earlier treatment could: (i) avoid or delay the progression to $\mathrm{MM}$; (ii) prevent the severe complications of end-organ damage; and (iii) potentially cure at least a proportion of patients with SMM. Thus, "to treat or not to treat" SMM, or even better, "are there patients with SMM who would benefit more from early treatment?" remains a controversial issue..$^{4,10-15}$

\section{Methodology}

Here, a European Myeloma Network (EMN) Expert Panel updates 2016 European perspectives on SMM, ${ }^{4}$ addressing current biological knowledge, new prognostic scoring systems and recent results of clinical trials, as well as providing practical recommendations. During two EMN Trialist meetings in 2019 and 2020, the areas of major concern in the management of SMM were selected by generating and rank-ordering key questions using the criterion of clinical relevance. Multistep procedures were utilized to achieve a consensus on recommendations. One panelist drafted the statements addressing the identified key questions. Subsequently, each panelist expressed his or her agreement with those statements and provided suggestions. The Delphi questionnaire method was used and a consensus of at least $80 \%$ was reached for all six final statements (median 93\%; range, $83-100 \%)$.

\section{New insights into the molecular pathogenesis of smoldering multiple myeloma}

All cases of MM evolve through MGUS and SMM stages, although these are often not clinically evident. ${ }^{16}$ The disease pathogenesis starts from intrinsic genomic defects in plasma cells, mainly translocations of oncogenes under the control of regulatory elements of the immunoglobulin heavy chain (IGH) locus or multiple trisomies of odd-numbered chromosomes. ${ }^{17}$ However, these are not sufficient to cause the progression from MGUS to SMM or MM, as in most cases the clone will not evolve in a patient's lifetime. Furthermore, high-risk translocations such as $t(4 ; 14)$ and $t(14 ; 16)$ are less frequent in asymptomatic cases, while $t(11 ; 14)$ is more frequent in SMM ${ }^{18}$ These different frequencies reflect different intrinsic propensities of the oncogenic translocation to drive symptomatic progression. Hyperdiploidy seems to slightly increase the risk of transformation to active $\mathrm{MM}$ as well. ${ }^{19}$ Interestingly, the number of chromosomal trisomies is lower in hyperdiploid MGUS than in hyperdiploid SMM ${ }^{20,21}$ suggesting that ongoing acquisition of genomic lesions may be the cause of progression. Consistently, secondary copy-number abnormalities, such as $\operatorname{del}(1 p), \operatorname{amp}(1 q), \operatorname{del}(16 q)$, and $\operatorname{del}(17 p)^{18,22}$ are also more frequent in MM than in SMM. Next-generation sequencing studies evidenced a globally lower number of mutations in MGUS and SMM than in MM. ${ }^{23,24}$ This was particularly true for mutations in KRAS, NRAS, FAM46C, but also for genes in the NF- $\mathrm{kB}$ pathway and DNA repair pathway genes. ${ }^{18}$ Conversely, high-risk SMM showed a landscape of mutations and chromosomal abnormalities more similar to that of $\mathrm{MM}^{25}$ Indeed, single-cell studies have highlighted, within a clonal plasma cell population, the presence of subclones with distinct phenotypes that could be linked to malignant progression and prove the step-wise evolution of MM. ${ }^{26}$

The analyses of paired SMM-MM genomes highlighted two patterns of progression: (i) evolution from minor or entirely new subclones, and (ii) no association with genomic changes. ${ }^{18,27-29}$ The former includes true asymptomatic cases that need to acquire new lesions to shift their clinical behavior towards an aggressive phenotype. The latter are aggressive cases just about to meet clinical criteria for progression, with generally a shorter time to evolution. ${ }^{25}$ Indeed, accumulating evidence suggests that changes in clonal substructure can be used to monitor SMM before end-organ damage develops. ${ }^{18,30}$ Genomic events associated with SMM progression include translo- 
cations between the IGH locus and the MYC oncogene $^{25,30,31}$ and accumulation of complex rearrangements. ${ }^{25,30,32}$ Last, the activity of mutational processes is different in MGUS/SMM and MM. Early mutations, acquired at the time of initiation, are caused by the DNA deaminase AID or from processes associated with cell aging. Late mutations, developing at the time of progression, arise from aberrant activity of the APOBEC family of cytidine deaminases, whose activity is absent in normal plasma cells. ${ }^{33-34}$

In clinical practice, recurrent translocations or hyperdiploidy can be assessed by fluorescence in situ hybridization or multiplex ligation-dependent probe amplification. ${ }^{35}$ Next-generation sequencing offers a more comprehensive evaluation of genomic lesions, ${ }^{36}$ but this approach must still be considered investigational and not a current standard.

The progression of MM also depends on the tumor microenvironment. ${ }^{37,38}$ In particular, clonal plasma cells feed on proliferative and anti-apoptotic signals from stromal cells, including interleukin-6, insulin-like growth factor 1 and vascular endothelial growth factor. Osteoblasts and osteoclasts play opposing roles, with reduced osteoprotegerin secretion by osteoblasts and increased RANKL secretion by stromal cells promoting the activity of osteoclasts, which in turn secrete interleukin-6. In addition, progressive SMM is associated with increased neo-angiogenesis, in which MM-induced endothelial cells carry specific gene expression signatures associated with disease evolution. Furthermore, immune cells in the microenvironment are actively involved in $\mathrm{MM}$ progression. Innate and acquired immunity may prevent clonal plasma cell growth in early asymptomatic stages, ${ }^{39}$ as indirectly shown by the unrestrained growth of plasma cells from asymptomatic patients xenografted into immunocompromised mice. ${ }^{40}$ In addition, single-cell studies have shown how clonal plasma cells may shape a permissive immune environment already from the MGUS stage. In a continuous pattern, progression from SMM to MM is also associated with reduced MHC class II expression in $\mathrm{CD}_{14}{ }^{+}$monocytes, an increase of regulatory $\mathrm{T}$ cells, loss of memory cytotoxic cells with skewing towards effector cells with suppressed or anergic phenotype, and upregulated interferon signaling promoting immunosuppression and $\mathrm{MM}$ growth. ${ }^{41}$ While these find- ings are less likely to be translated soon into clinical applications, the possibility of harnessing the immune microenvironment to generate diagnostic tests to predict progression, or even therapeutic approaches to halt progression, is intriguing.

\section{Current diagnosis and monitoring}

SMM is currently diagnosed according to International Myeloma Working Group (IMWG) criteria, based on amount of $\mathrm{M}$-component, percentage of clonal bone marrow plasma cells (BMPC) infiltration and no evidence of end-organ damage (CRAB) or amyloidosis (Table 1). ${ }^{3}$ Updated IMWG criteria re-classified $10-15 \%$ of patients previously diagnosed as having $\mathrm{SMM}^{3}$ on the basis of new myeloma-defining events (biomarkers of malignancy: $>60 \%$ BMPC, free light chains [FLC] ratio $>100^{42}$ and $>1$ focal lesion with magnetic resonance imaging [MRI]), the so-called "SLIM CRAB" criteria, as "ultra-high-risk" SMM. These patients are considered eligible for full MM treatments, having a risk of progression of about $80 \%$ at 2 years.

The new IMWG criteria emphasize the role of imaging to risk-stratify SMM, with patients having two or more focal lesions $>5 \mathrm{~mm}$ detected by MRI qualifying for active treatments. ${ }^{43,44}$ In particular, the IMWG recommends sensitive low-dose whole-body CT for the staging of monoclonal gammopathies. ${ }^{45}$ This imaging technique was recently validated in a prospective study of 100 patients with SMM at different timepoints to identify early bone lesions related to $\mathrm{MM}$ evolution. ${ }^{46}$ If low-dose wholebody CT is negative, whole-body or spine and pelvis MRI, where possible, should be pursued, ${ }^{43}$ although the latter MRI protocol can miss around $10 \%$ of non-axial lesions. Given the spatial distribution of focal bone lesions, it has been recently suggested that the cutoff for the number of focal lesions should be adapted according to the MRI protocol used. ${ }^{47}{ }^{18} \mathrm{~F}$-fluorodeoxyglucose positron emission tomography (PET)-CT may be considered an appropriate alternative to low-dose whole-body CT. It is highly recommended to distinguish between SMM and active MM, if low-dose whole-body CT is negative and whole-body MRI is unavailable. ${ }^{48}$ In the case of uncertain or borderline lesions, radiological studies

Table 1. Current diagnostic criteria for monoclonal gammopathy of undetermined significance, smoldering multiple myeloma and multiple myeloma, according to International Myeloma Working Group criteria.

\begin{tabular}{ll} 
Disease & Criteria \\
MGUS & Serum MC (non IgM type) $<3 \mathrm{~g} / \mathrm{dL}$ and clonal BMPC $<10 \%$ \\
& $\begin{array}{l}\text { Absence of myeloma-defining events, such as end-organ damage (CRAB) }{ }^{*} \text {, or other biomarkers of malignancy (SLiM)**, } \\
\text { or amyloidosis, which can be attributed to the plasma cell proliferative disorder }\end{array}$ \\
\hline SMM & $\begin{array}{l}\text { Serum MC (IgG or IgA) }>3 \mathrm{~g} / \mathrm{dL} \text { or urinary MC }>500 \mathrm{mg} \text { per } 24 \text { hours and clonal BMPC } \geq 10 \% \text { and }<60 \% \\
\text { Absence of myeloma-defining events, such as end-organ damage (CRAB) }{ }^{*} \text {, or other biomarkers of malignancy (SLiM)*, } \\
\text { or amyloidosis, that can be attributed to the plasma cell proliferative disorder }\end{array}$ \\
\hline MM & $\begin{array}{l}\text { Clonal BMPC cells }>10 \% \text { or biopsy proven plasmacytoma and any one or more of myeloma-defining events, such as end-organ } \\
\text { damage (CRAB)*, or other biomarkers of malignancy (SLIM) }{ }^{*}, \text { or amyloidosis, which can be attributed to the plasma cell } \\
\text { proliferative disorder }\end{array}$ \\
\hline
\end{tabular}

MGUS: monoclonal gammopathy of undetermined significance; MC: M-component; BMPC: bone marrow plasma cells; SMM: smoldering multiple myeloma; MM: multiple myeloma. * CRAB: serum Calcium $>1 \mathrm{mg} / \mathrm{dL}$ above the upper limit of normal value or $>11 \mathrm{mg} / \mathrm{dL}$; Renal insufficiency: serum creatinine $>2 \mathrm{mg} / \mathrm{dL}$ or creatinine clearance $<40$ $\mathrm{mL} / \mathrm{min}$; Anemia: hemoglobin $>2 \mathrm{~g} / \mathrm{dL}$ below the lower limit of normal value or $10 \mathrm{~g} / \mathrm{dL}$; Bone lesions: one or more lytic lesions on skeletal radiography or computed tomography (including positron emission tomography). * ${ }^{*}$ SLiM: clonal bone marrow plasma cell percentage $\geq$ Sixty percent; serum involved/uninvolved free LIght chains ratio $>100$; Magnetic resonance imaging: more than one focal lesion $\geq 5 \mathrm{~mm}$. 
should be repeated, preferably alternating different techniques, every 3-6 months or whenever clinically indicated, until a definitive diagnosis of symptomatic MM is reasonably made or excluded, (i.e., the suspicious lesion remains stable, is not accompanied by other signs of progression, or is disappearing).

Clinical and laboratory monitoring of SMM should initially be performed every 2-3 months after diagnosis for 6-12 months. ${ }^{49}$ If test results are stable, patients may be followed every 4-6 months for another year and every 612 months thereafter. However, follow-up should be individualized based on risk of progression. Despite the absence of prospective data, according to the Expert Panel, imaging evaluation might preferably be repeated annually with MRI (because of the higher sensitivity for early damage) for the first 5 years (then stopped or the frequency reduced) or at clinical suspicion/pain or progressive increase of $\mathrm{M}$-component (low-dose whole-body CT or MRI). A detailed imaging algorithm for patients with SMM is reported in the recent IMWG consensus on imaging. ${ }^{43}$

Importantly, combined, longitudinal evaluation and review of all relevant disease parameters may be required to interpret dynamics of the disease correctly. ${ }^{4}$

\section{New prognostic scores}

During the last years, several risk scores for SMM progression, combining routinely used laboratory parameters, have emerged. $4,8,99$ These include the BMPC infiltration rate, an aberrant plasma cell phenotype (>95\% clonal BMPC within the BMPC compartment), immunoparesis, the amount of serum $\mathrm{M}$-component, an altered FLC ratio, and albumin levels. ${ }^{50-54}$

Further studies have highlighted the prognostic value of additional factors: (i) a progressive increase of the $M$ component and Bence-Jones proteinuria over time ${ }^{.55,56}$ (ii) an evolving decrease in hemoglobin, ${ }^{57}$ (iii) bone marrow biopsy characteristics, ${ }^{58}$ (iv) presence of circulating plasma cells and their proliferative activity in the bone marrow; ${ }^{59,60}(\mathrm{v})$ bone involvement detected by MRI or PET-CT

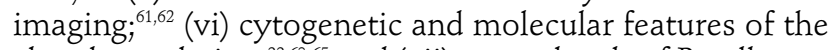
clonal population; ${ }^{22,63-65}$ and (vii) serum levels of B-cell maturation antigen. ${ }^{66}$ In particular, a Southwest Oncology Group model, incorporating serum FLC values, serum Mcomponent and the University of Arkansas Medical Sciences 70-gene expression profile signature (GEP-70), predicted 2-year progression rates of $66.7 \%, 21.9 \%$ and $3.4 \%$ in patients with two or three, one, and no risk factors, respectively, ranking statistically first among other investigated clinical risk scores available at the time of analysis (Table 2). ${ }^{67}$

Since ultra-high-risk SMM patients are now considered to have $\mathrm{MM}^{3}$, previously defined risk stratification models need to be re-assessed. Researchers at Mayo Clinic reexamined their initial cohort of 421 patients with SMM who met the 2014 IMWG criteria to re-classify risk factors for progression. ${ }^{6}$ The median time to progression to symptomatic MM was 57 months. Based on multivariate analysis data, a new Mayo risk model was proposed utilizing the same three parameters previously identified in 2008 , but with different cut-offs: involved to uninvolved serum FLC ratio $>20$, serum $\mathrm{M}$-component $>2 \mathrm{~g} / \mathrm{dL}$, and BMPC infiltration $>20 \%(20 / 2 / 20$ SMM score, Table 2).
On this basis, three risk groups were identified: low-risk: no risk factors; intermediate risk: one risk factor; highrisk: two or more risk factors. The estimated median times to progression were 109.8 months, 67.8 months and 29.2 months, respectively. Criticisms of this model, however, could be that the cut-offs were developed due to best subgroup separations, the entire cohort and subgroups were limited, and a validation analysis was not available at the time of publication.

A panel of IMWG experts recently conducted a larger, multicenter, retrospective study of 1,996 SMM patients diagnosed according to the 2014 IMWG criteria, to develop a robust risk stratification model ${ }^{69}$ (Table 2$)$. The follow-up from diagnosis was 3 years. The median time to progression of the entire cohort was 6.4 years, while the 2-, 5-, and 10-year risks of progression were $22 \%$, $42 \%$ and $64 \%$, respectively. Stepwise selection and multivariable analysis confirmed the value of $20 / 2 / 20$ parameters and 1,363 patients with all three factors available were stratified in the same three categories, whose 2 year progression rates were $6 \%, 18 \%$ and $44 \%$, respectively. Additional analyses were conducted in 689 patients with a complete dataset by adding the presence of at least one of the following recurrent cytogenetic abnormalities: $t(4 ; 14), t(14 ; 16), 1 q$ gain, and del13q. Pooled data identified four risk categories, corresponding with 2 -year risks of progression of $6 \%, 23 \%, 37 \%$, and $63 \%$ (Table 2 ). To define a scoring tool providing a more individualized risk assessment, the three original risk factors (involved to uninvolved serum FLC ratio, serum $M$ component, and BMPC infiltration), together with cytogenetic abnormalities, were included in a new logistic regression model based on the entire range of values instead of using single cut points (Table 2). Using this approach, the 2 -year risks of progression were $3.8 \%$ in patients with a total score of $0-4,26 \%$ in those with a score $5-8,51 \%$ in those with a score $9-12$, and $73 \%$ when the score was $>12$.

The Czech Myeloma Group recently developed a simplified alternative model for SMM based exclusively on different serum parameters ${ }^{70}$ (Table 2). Data were collected from a training group of 287 patients and validated in an independent cohort of 240 patients. With a median follow-up of $2.4 / 2.5$ years in the two groups, progression to MM occurred in $52 \%$ and $39 \%$ of patients, respectively. The median risks of progression per year were $11 \%$ and $10 \%$, during the first 5 years after diagnosis, respectively. A serum FLC ratio of $>30$, immunoparesis (at least one uninvolved immunoglobulin below reference levels), and serum $\mathrm{M}$-component $\geq 2.3 \mathrm{~g} / \mathrm{dL}$ emerged as predictors of 2 -year progression rate in a combined multivariate analysis. Based on these parameters, a new risk model was proposed with four groups of patients defined by the presence of none, one, two or three of these risk factors. Notably, the 2-year risks of progression in the two cohorts were $79 \%$ and $80 \%$ for those patients with three risk factors.

In a recently proposed "genomic model", next-generation sequencing was applied in a retrospective cohort of 214 patients with SMM, with whole exome sequencing performed on 166 tumors, and deep targeted sequencing on 48 tumors $^{71}$ (Table 2). The model incorporated information on DNA repair/MAPK pathway gene alterations and MYC aberrations. All these genomic abnormalities independently predicted progression after accounting for 
Table 2. Most recent prognostic models for smoldering multiple myeloma.

\begin{tabular}{|c|c|c|c|c|}
\hline Model & Risk factors & Risk Group & $\begin{array}{l}\text { 2-year } \\
\text { PD rate (\%) }\end{array}$ & $\begin{array}{l}\text { Median TIP } \\
\text { (months) }\end{array}$ \\
\hline SWOG 2014 & $\mathrm{MC}>3 \mathrm{~g} / \mathrm{dL}, \mathrm{sFLC}>25 \mathrm{mg} / \mathrm{dL}, \mathrm{GEP}-70>0.26$ & $\begin{array}{l}\text { Low ( } 0 \text { factors), } \mathrm{n}=60 \\
\text { Intermediate ( } 1 \text { factor), } \mathrm{n}=39 \\
\text { High ( } \geq 2 \text { factors), } \mathrm{n}=18\end{array}$ & $\begin{array}{c}3.4 \\
21.9 \\
66.7\end{array}$ & \\
\hline Mayo $2018^{68}$ & $s F L C r>20, \mathrm{MC}>2 \mathrm{~g} / \mathrm{dL}, \mathrm{BMPC}>20 \%$ & $\begin{array}{l}\text { Low ( } 0 \text { factors), } n=143 \\
\text { Intermediate ( } 1 \text { factor), } n=121 \\
\text { High ( } \geq 2 \text { factors), } n=153\end{array}$ & $\begin{array}{c}9.7 \\
26.3 \\
47.4\end{array}$ & $\begin{array}{l}109.8 \\
67.8 \\
29.2\end{array}$ \\
\hline \multirow[t]{2}{*}{ IMWG $2020^{69}$} & $\mathrm{MC}>2 \mathrm{~g} / \mathrm{dL}, \mathrm{sFLCr}>20, \mathrm{BMPC}>20 \%$ & $\begin{array}{l}\text { Low ( } 0 \text { factors), } n=522 \\
\text { Intermediate }(1 \text { factor }), n=445 \\
\text { High ( } \geq 2 \text { factors, } n=396\end{array}$ & $\begin{array}{c}6 \\
18 \\
44\end{array}$ & \\
\hline & $\begin{array}{l}\text { + high risk cytogenetics: }[\mathrm{t}(4 ; 14), \mathrm{t}(14 ; 16) \\
+1 \mathrm{q}, \operatorname{del}(13 \mathrm{q})]\end{array}$ & $\begin{array}{l}\text { Low }(0 \text { factors/score } 0-4) *, n=241 \\
\text { Low-intermediate }(1 \text { factor/score } 5-8), n=264 \\
\text { Intermediate }(2 \text { factors/score } 9-12), n=233 \\
\text { High }(3-4 \text { factors/score }>12), n=51\end{array}$ & $\begin{array}{c}6 / 3.8 \\
22 / 26.2 \\
45.5 / 51.1 \\
63.1 / 72.5\end{array}$ & \\
\hline CMG $2020^{70}$ & $\begin{array}{l}\text { Immunoparesis (at least one uninvolved } \\
\text { immunoglobulin below reference levels), } \\
\text { sFLCr }>30, \mathrm{MC} \geq 2.3 \mathrm{~g} / \mathrm{dL}\end{array}$ & $\begin{array}{l}\text { Low ( } 0 \text { factors), } n=48 / 26^{* *} \\
\text { Intermediate }(1 \text { factor }), n=44 / 34 \\
\text { High ( } 2 \text { factors), } n=32 / 41 \\
\text { Very high ( } 3 \text { factors), } n=15 / 12\end{array}$ & $\begin{array}{c}8.5 / 5.3 * * \\
20.9 / 7.5 \\
41.9 / 44.8 \\
78.7 / 81.3\end{array}$ & \\
\hline $\begin{array}{l}\text { Dana Farber } \\
2020^{71}\end{array}$ & $\begin{array}{l}\text { DNA repair pathway gene alterations [mutations } \\
\text { in TP53 and } A T M \text {, del(17p)], MAPK pathway gene } \\
\text { mutations (KRAS, NRAS), MYC aberrations } \\
\text { (translocations or copy-number variations). }\end{array}$ & $\begin{array}{l}0 \text { factors, } n=58 \\
\text { At least } 1 \text { factor, } n=24\end{array}$ & $\begin{array}{l}86.4 \\
14.4\end{array}$ & \\
\hline
\end{tabular}

PD: progressive disease; TTP: time to progression; SWOG; Southwest Oncology Group; MC: monoclonal component; sFLC: serum free light chains; sFLCr: serum free light chain ratio; BMPC: bone marrow plasma cells; GEP-70: University of Arkansas Medical Sciences 70-gene expression profile signature; CMG: Czech Myeloma Group. *SFCLr score: <10: 0; 10-25: 2;>25-40: 3; >40: 5 . MC score: 0-1.5 g/dL: 0; $>1.5-3$ g/dL: $3 ;>3$ g/dL: 4. BMPC score: 0-15\%: 0; $>15-20 \%: 2 ;>20-30 \%: 3 ;>30-40 \%: 5 ;>40 \%: 6$. ** Validation cohort.

clinical risk staging. Patients without such alterations had a median time to progression of 7.2 years versus 1.2 in those with one or more alterations. In addition, the risk was cumulative, and patients with two or more alterations progressed the fastest. These results were validated in an external cohort of 72 patients with SMM previously sequenced. Importantly, this model outperformed the Mayo Clinic 2008 and 2018 prediction models.

\section{Early treatment}

Possible treatments of SMM vary considerably, with aims ranging from disease control, to delaying progression, and ideally cure (Table 3 ). Eight randomized, controlled trials covering 885 patients were evaluated in a recent meta-analysis that compared early versus deferred treatment in SMM. ${ }^{72}$ These studies included patients treated with melphalan + prednisone, ${ }^{73-75}$ bisphosphonates \pm thalidomide ${ }^{76-78}$ siltuximab ${ }^{79}$ and lenalidomide + dexamethasone. ${ }^{80,81}$ Overall, early treatment significantly decreased progression of SMM (risk ratio [RR] $=0.53$, $95 \%$ confidence interval $[95 \% \mathrm{CI}]: 0.33-0.87, P=0.01)$, particularly in patients receiving melphalan + prednisone $(\mathrm{RR}=0.22$, 95\% CI: $0.08-0.64, P=0.005)$ or immunomodulatory drugs $(\mathrm{RR}=0.43,95 \% \mathrm{CI}: 0.31-0.59, P<0.00001)$, and in the high-risk SMM subgroup ( $R R=0.51,95 \% \mathrm{CI}$ : $0.37-0.70, P=0.0001)$. In the latter patients, treatment of SMM also significantly decreased mortality $(R R=0.53$, 95\% CI: 0.29-0.96, $P=0.04)$. Regarding the most relevant adverse events, the risk of secondary primary malignancies was significantly increased with early treatment $(\mathrm{RR}=4.13$, 95\% CI: 1.07-15.97, $P=0.04)$. Major criticisms to this meta-analysis were the wide heterogeneity of therapeutic approaches used and the lack of risk stratification in all but one trial.

\section{Treatment options with novel agents}

\section{Lenalidomide}

The results of two phase III, prospective studies have been fully published, providing support to the use of lenalidomide ( \pm dexamethasone) in patients with highrisk SMM.

The Spanish QuiRedex phase III trial (NCT00480363) was a pivotal study conducted in 119 patients with highrisk SMM, defined by the presence of both BMPC $>10 \%$ and $\mathrm{M}$-component $>3 \mathrm{~g} / \mathrm{dL}$ or, if only one criterion was present, patients had to have $<95 \%$ aberrant BMPC by immunophenotyping plus immunoparesis. Patients were randomized to receive nine 4 -week induction cycles with lenalidomide + dexamethasone followed by maintenance with lenalidomide alone for 2 years or to undergo observation. ${ }^{80,81}$ The primary endpoint was time to progression. Updated results after a median follow-up of 10.8 years revealed a $46 \%$ reduction in the risk of death (hazard ratio $[\mathrm{HR}]=0.54 ; 95 \% \mathrm{CI}: 0.3-0.9 ; P=0.034$ ) and $73 \%$ in that of progression (HR=0.27; 95\% CI: $0.16-0.42$; $P<0.0001)$ for early treatment versus observation. ${ }^{82}$ The median overall survival had not been reached in the treatment arm, while it was 7.8 years in the control arm. The updated median time to progression was 9.0 versus 2.1 years in patients receiving treatment and in the control group, respectively. No differences were observed between arms when overall survival was compared from the start of subsequent therapy in patients who progressed to active MM, suggesting that, once the patient has progressed, lenalidomide treatment would not induce the appearance of resistant clones. The frequency of secondary primary malignancies was higher in the treatment group than in the observation group (10\% vs. $2 \%)$, but the cumulative risk did not differ significantly $(P=0.07)$. Extensive phenotypic studies suggested that 
Table 3. Update of clinical trials in smoldering multiple myeloma with currently available results. Other ongoing studies without published results are reported in the text of the paper.

\begin{tabular}{|c|c|c|c|c|c|c|}
\hline & $\begin{array}{l}\text { Phase, n, of } \\
\text { patients }\end{array}$ & Treatments & Risk stratification & $\begin{array}{l}\text { Primary endpoint, } \\
\text { FU }\end{array}$ & ORR, CR, MRD & PFS, TIP, OS \\
\hline $\begin{array}{l}\text { QuiRedex } \\
\text { NCT00480363 } \\
{[80-82]}\end{array}$ & III, n=119 & $\begin{array}{l}\text { Induction: } 28 \text {-day cycle } \\
\text { [C1-9] } \\
\text { Lenalidomide } 25 \mathrm{mg} \\
\text { p.o. days } 1-21+\text { Dex } 20 \mathrm{mg} \\
\text { p.o. days } 1-4,12-15 ; \\
\text { Maintenance: } 28 \text {-day cycle } \\
\text { [C1-24] Lenalidomide } \\
10 \text { mg p.o days } 1-21(\mathrm{n}=57) \\
\text { Observation }(\mathrm{n}=62)\end{array}$ & $\begin{array}{l}\text { High-risk by BMPC }>10 \% \\
\text { and } \mathrm{MC}>3 \mathrm{~g} / \mathrm{dL} \text { or, } \\
\text { if only one criterion } \\
\text { present, BMPC } \\
\text { with aberrant phenotype } \\
>95 \% \text { plus immunoparesis }\end{array}$ & $\begin{array}{l}\text { TTP } \\
\text { Median FU: } 10.8 \text { years }\end{array}$ & $\begin{array}{l}\text { After induction: } \\
\text { ORR 79\%, CR: 14\% } \\
\text { After maintenance: } \\
\text { ORR 90\%, CR 26\% } \\
\text { ORR/CR: 0\% }\end{array}$ & $\begin{array}{l}\text { Median TTP: } \\
9.0 \text { vs. } 2.1 \text { years } \\
(P=0.034) \\
\text { Median OS: } \\
\text { NR vs. } 7.8 \text { years } \\
(P<0.0001)\end{array}$ \\
\hline $\begin{array}{l}\text { SWOG E3A06 } \\
\text { NCT01169337 } \\
{[84]}\end{array}$ & III, $n=182$ & $\begin{array}{l}\text { Continuous therapy: } \\
28 \text { day-cycle }[\mathrm{Cl}-\mathrm{PD}] \text { : } \\
\text { Lenalidomide } 25 \mathrm{mg} \\
\text { p.o. days } 1-21 \\
(\mathrm{n}=90) \\
\text { Observation }(\mathrm{n}=92)\end{array}$ & $\begin{array}{l}\text { Intermediate or high-risk } \\
\text { by BMPC }>10 \% \text {, or sheets } \\
\text { and FLC ratio }<0.26 \\
\text { or }>1.65\end{array}$ & $\begin{array}{l}\text { PFS } \\
\text { Median FU: } \\
35 \text { months }\end{array}$ & ORR 50\%, CR 0\% & $\begin{array}{l}\text { 3-year PFS: } \\
91 \% \text { vs. } 66 \% \\
(P=0.002)\end{array}$ \\
\hline $\begin{array}{l}\text { NCT01572480 } \\
{[23,85]}\end{array}$ & $\mathrm{II}, \mathrm{n}=18$ & $\begin{array}{l}\text { Induction: 28-day cycle } \\
\text { [C1-8]: Carfilzomib } \\
\text { 20/36 mg/m² i.v. } \\
\text { days 1,2,8,9,15, } 16+ \\
\text { Lenalidomide } 25 \mathrm{mg} \\
\text { p.o. days 1-21 + Dex }\end{array}$ & $\begin{array}{l}\text { High-risk by PETHEMA } \\
\text { and MAYO } 2008 \text { criteria }\end{array}$ & $\begin{array}{l}\text { ORR, MRD } \\
\text { Median FU: } \\
43.3 \text { months }\end{array}$ & $\begin{array}{l}\text { ORR } 100 \% \text {, } \\
\text { MRD negativity: } \\
92 \% \text { by MFC, } \\
75 \% \text { by NGS, }\end{array}$ & $\begin{array}{l}\text { Estimated } \\
\text { 4-year PFS: 71\% } \\
\text { Estimated } \\
\text { 4-year OS: 100\% }\end{array}$ \\
\hline
\end{tabular}

(C5-8) p.o or iv.

days $1,2,8,9,15,16$

Maintenance: 28-day cycle

[C1 - 24] Lenalidomide

$25 \mathrm{mg}$ days 1-21

\begin{tabular}{|c|c|c|c|c|c|c|}
\hline $\begin{array}{l}\text { GEM-CESAR } \\
\text { NCT02415413 } \\
{[87,88]}\end{array}$ & III, $\mathrm{n}=90$ & $\begin{array}{l}\text { Induction: } 28 \text {-day-cycle } \\
\text { [C1-6]: Carfilzomib } \\
\text { 20/36 } \mathrm{mg} / \mathrm{m}^{2} \text { i.v. } \\
\text { days 1,2, 8, 9, } 15,16 \\
\text { + Lenalidomide } 25 \mathrm{mg} \\
\text { p.o. days } 1-21+\text { Dex } \\
40 \mathrm{mg} \text { days } 1,8,15,22 \\
\text { AuSCT: Melphalan } 200 \mathrm{mg} / \mathrm{m}^{2} \\
\text { Consolidation: KRd } \\
\text { as induction for } 2 \text { cycles } \\
\text { Maintenance: } 28 \text { day-cycle } \\
\text { Lenalidomide } 10 \mathrm{mg} \\
\text { days } 1-21+\text { Dex } 20 \mathrm{mg} \\
\text { days } 1,8,15,22 \text { for } 2 \text { years }\end{array}$ & $\begin{array}{l}\text { High-risk by PETHEMA } \\
\text { criteria (ultra-high risk } \\
\text { patients were included, } \\
\text { ref. 3) }\end{array}$ & $\begin{array}{l}\text { MRD } \\
\text { Median FU: } \\
32 \text { months }\end{array}$ & $\begin{array}{l}\text { ORR: } 98 \% \\
\text { post-induction, } \\
98 \% \text { post-AuSCT, } 100 \% \\
\text { post-consolidation } \\
\text { and maintenance; } \\
\geq \text { CR: } 38.4 \%, 61.5 \% \\
\text { and } 68.6 \% \text { at the same } \\
\text { time-points } \\
\text { MRD negativity } 23 \% \text {, } \\
44 \% \text { and } 55 \% \text { at the } \\
\text { same time points }\end{array}$ & $\begin{array}{l}\text { PFS 93\% } \\
\text { ( } 5 \text { biochemical } \\
\text { progressions) }\end{array}$ \\
\hline $\begin{array}{l}\text { NCT02697383 } \\
\text { [89] }\end{array}$ & $\begin{array}{l}\text { Pilot study, } \\
\mathrm{n}=14\end{array}$ & $\begin{array}{l}\text { Induction: 28-day cycle } \\
\text { [C1-12] Ixazomib } 4 \mathrm{mg} \text { p.o. } \\
\text { days 1,8, } 15+\text { Dex } 40 \mathrm{mg} \\
\text { p.o. days } 1,8,15,22 \\
\text { Maintenance: 28-day cycle } \\
\text { [C1-24] Ixazomib } 4 \text { mg p.o. } \\
\text { days } 1,8,15\end{array}$ & $\begin{array}{l}\text { High-risk by PETHEMA } \\
\text { or Mayo } 2008 \text { criteria }\end{array}$ & $\begin{array}{l}\text { ORR } \\
\text { Median FU } 17 \text { months }\end{array}$ & ORR 64\% (no CR) & $\begin{array}{l}\text { No progression } \\
\text { to MM }\end{array}$ \\
\hline $\begin{array}{l}\text { NCT02916771 } \\
{[90]}\end{array}$ & $\begin{array}{l}\mathrm{II}, \mathrm{n}=26 \\
(56 \text { planned })\end{array}$ & $\begin{array}{l}\text { Induction: 28-day-cycle } \\
\text { [C1-9]: Ixazomib } 4 \mathrm{mg} \\
\text { p.o. days } 1,8,15+ \\
\text { Lenalidomide } 25 \mathrm{mg} \text { p.o. } \\
\text { days } 1-21+\text { Dex } 40 \mathrm{mg} \\
\text { p.o. days } 1,8,15,22 \\
\text { Maintenance: } 28 \text {-day cycle } \\
\text { [C10-24]: Ixazomib } 4 \text { mg p.o. } \\
\text { days 1,8, } 15 \text { + Lenalidomide } \\
\text { 15 mg p.o. days 1-21 }(\mathrm{n}=45)\end{array}$ & High risk, (ref. 8) & $\begin{array}{l}\text { PFS } \\
\text { Median number } \\
\text { of cycles: } 8\end{array}$ & ORR 89\%, CR 19.2\% & $\begin{array}{l}\text { No progression } \\
\text { to MM }\end{array}$ \\
\hline $\begin{array}{l}\text { CENTAURUS } \\
\text { NCT02316106 } \\
{[92]}\end{array}$ & II, $n=123$ & $\begin{array}{l}\text { Daratumumab } 16 \mathrm{mg} / \mathrm{kg} \text { i.v. } \\
\text { in 8-week cycles: } \\
\text { Extended intense }(\mathrm{n}=41) \text { : } \\
\text { [C1] every } 1 \text { week; [C2-3] }\end{array}$ & $\begin{array}{l}\text { Intermediate or high-risk } \\
\text { by BMPC } \geq 10 \% \text { and }<60 \% \\
\text { and at least one of } \\
\text { the following: MC }\end{array}$ & $\begin{array}{l}\text { CR and PD/DR ratio } \\
\text { Median FU } 26 \text { months }\end{array}$ & $\begin{array}{l}\text { ORR 56\%, CR 4.9\%, } \\
\text { PD/DR } 0.059\end{array}$ & $\begin{array}{l}\text { 2-year PFS: } 89.9 \% \\
\text { extended intense, } \\
\text { vs. } 82.0 \% \\
\text { intermediate }\end{array}$ \\
\hline
\end{tabular}


every other week; [C4-7]

every 4 weeks;

[C8-20] every 8 weeks

Intermediate intense $(n=41)$

[C1] every 1 week and

[C2-20] every 8 weeks

Short dosing $(\mathrm{n}=41)$ : $[\mathrm{Cl}]$ every

1 week

\begin{tabular}{|c|c|c|}
\hline $\begin{array}{l}\text { NCT02960555 } \\
{[96]}\end{array}$ & $\begin{array}{l}\text { II, } \mathrm{n}=24 \\
\text { (planned } \\
\text { n. 61) }\end{array}$ & $\begin{array}{l}\text { Isatuximab } 20 \mathrm{mg} / \mathrm{kg} \text { i.v. } \\
\text { in } 4 \text { week cycle }[\mathrm{Cl}] \text { every } \\
1 \text { week; [C2-6] every other } \\
\text { week; [C7-30] every } 4 \text { weeks }\end{array}$ \\
\hline $\begin{array}{l}\text { NT01441973 } \\
{[97]}\end{array}$ & II, $\mathrm{n}=31$ & $\begin{array}{l}\text { Elotuzumab } 20 \mathrm{mg} / \mathrm{kg} \text { i.v. } \\
\text { [C1] days } 1,8 \text {, then } \\
\text { [C2-progressive disease] } \\
\text { monthly every } 4 \text { weeks }\end{array}$ \\
\hline
\end{tabular}

Elotuzumab $10 \mathrm{mg} / \mathrm{kg}$ i.v.

[C1] days $1,8,15,22$, then

[C2- progressive disease]

monthly every 2 weeks

\begin{tabular}{|c|c|c|c|c|c|c|}
\hline $\begin{array}{l}\text { NCT02279394 } \\
{[98]}\end{array}$ & II, $\mathrm{n}=50$ & $\begin{array}{l}\text { Induction: 28-day cycle } \\
\text { [C1-2] Elotuzumab } 10 \mathrm{mg} / \mathrm{kg} \\
\text { i.v. days } 1,8,15,22+ \\
\text { Lenalidomide } 25 \mathrm{mg} \text { p.o. days } \\
\text { 1-21 + Dex } 40 \mathrm{mg} \text { p.o. } \\
\text { days } 1,8,15,22 \\
\text { [C3-8]: Stem cell collection; } \\
\text { Elotuzumab } 10 \mathrm{mg} / \mathrm{kg} \text { i.v. } \\
\text { days } 1,15+\text { Lenalidomide } \\
\text { as } 25 \mathrm{mg} \text { p.o. days } 1-21+ \\
\text { Dex } 40 \mathrm{mg} \text { p.o. days } 1,8,15 \\
\text { Maintenance: } 28 \text {-day cycle } \\
\text { [C9-24] Elotuzumab } 10 \mathrm{mg} / \mathrm{kg} \\
\text { i.v. days 1 + Lenalidomide } 25 \mathrm{mg} \\
\text { p.o. days 1-21 }\end{array}$ & High-risk, (ref. 8) & $\begin{array}{l}\text { PFS } \\
\text { FU NA }\end{array}$ & ORR 84\%, CR 6\% & $\begin{array}{l}\text { No progression } \\
\text { to MM }\end{array}$ \\
\hline $\begin{array}{l}\text { NCT02603887 } \\
\text { [99] }\end{array}$ & $\begin{array}{l}\text { Pilot study, } \\
\mathrm{n}=13\end{array}$ & $\begin{array}{l}\text { Pembrolizumab } 200 \text { mg i.v. } \\
\text { every } 3 \text { weeks } \times 8 \text { cycles; } \\
\text { with option to continue up } \\
\text { to } 24 \text { cycles if continued } \\
\text { benefit }\end{array}$ & $\begin{array}{l}\text { Intermediate to high risk } \\
\text { by either PETHEMA, Mayo } \\
2008 \text { or SWOG criteria }\end{array}$ & $\begin{array}{l}\text { ORR } \\
\text { Median number } \\
\text { of cycles: } 8\end{array}$ & $\begin{array}{l}\text { ORR 8\%, CR 8\%, } \\
\text { MRD negativity 8\% }\end{array}$ & $\begin{array}{l}15 \% \text { of patients } \\
\text { progressed } \\
\text { to MM }\end{array}$ \\
\hline $\begin{array}{l}\text { NCT01484275 } \\
\text { [79] }\end{array}$ & III, $n=85$ & $\begin{array}{l}\text { Siltuximab } 15 \mathrm{mg} / \mathrm{kg} \text { i.v. in } \\
2 \text { h every } 4 \text { weeks ( }=43) \\
\text { [C1-progressive disease] } \\
(\mathrm{n}=43)\end{array}$ & $\begin{array}{l}\text { High risk by } \mathrm{BMPC}>10 \% \\
\text { and either } \mathrm{MC}>3 \mathrm{~g} / \mathrm{dL} \text {, or } \\
\text { FLC ratio }<0.126 />8 \\
\text { and } \mathrm{MC}>1 /<3 \mathrm{~g} / \mathrm{dL} \\
\text { (32\% ultra-high risk; ref } 3 \text { ) }\end{array}$ & $\begin{array}{l}\text { PFS } \\
\text { Median FU } \\
29.2 \text { months }\end{array}$ & NA & $\begin{array}{l}\text { 1-year PFS: } 84.5 \% \\
\text { siltuximab } \\
\text { vs. } 74.4 \% \\
\text { observation } \\
(P<0.06) \\
\text { Median PFS: NR } \\
\text { siltuximab vs.: } \\
23.5 \text { months } \\
\text { observation } \\
\text { OS not reached } \\
\text { in both arms }\end{array}$ \\
\hline $\begin{array}{l}\text { NCT01718899 } \\
{[100]}\end{array}$ & I-IIa, $n=20$ & 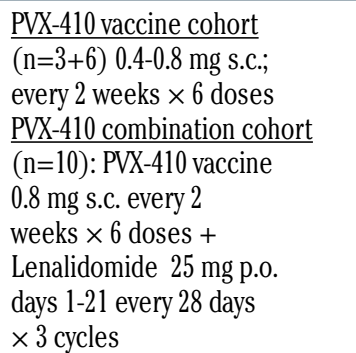 & $\begin{array}{l}\text { Moderate to high risk by } \\
\mathrm{MC} \geq 3 \mathrm{~g} / \mathrm{dL}, \mathrm{BMPC}>10 \% \text {, } \\
\text { abnormal FLC ratio } \\
(0.26-1.65) \\
\text { Moderate (2 risk factors) } \\
\text { or high risk (3 risk factors) }\end{array}$ & $\begin{array}{l}\text { Safety and immune } \\
\text { response }\end{array}$ & $\begin{array}{l}\text { Immune response } \\
\text { 95\%; (10/11 PVX-410 } \\
\text { monotherapy, } \\
\text { 9/9 PVX-410 } \\
\text { combination) }\end{array}$ & $\begin{array}{l}\text { PVX-410-alone: } \\
3 \text { progressions } \\
\text { to MM (median } \\
\text { TTP } 36 \text { weeks) } \\
\\
\text { Combination } \\
\text { cohort: } \\
1 \text { progression } \\
\text { to MM, median } \\
\text { TTP NR }\end{array}$ \\
\hline
\end{tabular}

FU: follow-up, ORR: overall response rate; CR: complete remission; MRD: minimal residual disease,PFS: progression-free survival;TTP: time to progression; OS: overall survival; C: cycle; p.o.: per os; i.v. intravenous; s.c.: subcutaneous; BMPC: bone marrow plasma cells; MC: M-component; KRd: carfilzomib, lenalidomide, dexamethasone; NR: not reached; FLC: free light chains; Dex: dexamethasone; MFC: multicolor flow cytometry; NGS: next generation sequencing; AuSCT: autologous stem cell transplantation; NA, not available; NK: natural killer; MM: multiple myeloma; PD/DR: progressive disease/death rate per patient-year ratio; Mayo 2008: ref. 51; PETHEMA: ref. 50; SWOG: ref. 66. $\geq 3 \mathrm{~g} / \mathrm{dL}(\operatorname{IgA} \geq 2 \mathrm{~g} / \mathrm{dl})$, urine $\mathrm{MC}>500 \mathrm{mg} / 24 \mathrm{~h}$, FLC ratio $<0.126$ or $>8$

High-risk (criteria NA)

High risk by $\mathrm{MC} \geq 3 \mathrm{~g} / \mathrm{dL}$ with $\mathrm{BMPC} \geq 10 \%$; or MC $1-3 \mathrm{~g} / \mathrm{dL}$ (alternatively urine $\mathrm{MC}>200 \mathrm{mg} / 24 \mathrm{~h}$ ), $\mathrm{BMPC} \geq 10 \%$ and FLC ratio $<0.125$ or $>8.0$

\section{ORR}

PD/DR 0.150

Median number of cycles: 11.5

\section{Relationship CD $56^{\mathrm{dim}}$}

NK cells and $\mathrm{MC}$ protein reduction (not found)

FU at least 28 months

ORR 64\%, CR 5\%, with MRD negativity

ORR $10 \%$
(cumulative) $\quad \begin{aligned} & \text { 2-year PFS } 69 \% \\ & \text { (cumulative) }\end{aligned}$

\section{ORR 54\%, CR 9.8\%,} PD/DR 0.107

intense vs. 75.3\% short dosing 
the impaired immune system of high-risk SMM patients could be reactivated by the immunomodulatory activity of lenalidomide. ${ }^{33}$

The Eastern Cooperative Oncology Group E3A06 phase III trial (NCT01169337) assessed the efficacy of lenalidomide monotherapy compared with observation in patients with intermediate- or high-risk SMM. ${ }^{80}$ Lenalidomide was administered orally as a single agent until disease progression, toxicity, or withdrawal for other reasons. Response to therapy was observed in $50 \%$ of patients in the lenalidomide arm, with no responses in the observation arm. With a median follow-up of 35 months, progression-free survival (the primary endpoint of the study) was significantly longer with lenalidomide than with observation ( $\mathrm{HR}=0.28$; $95 \% \mathrm{CI}$ : $0.12-0.62 ; P=0.002)$, indicating a $72 \%$ decrease in the risk of progression. The most pronounced and significant benefit was seen in patients with high-risk SMM, as defined by both 2008 and 2018 Mayo Clinic risk models. Six deaths occurred, two in the lenalidomide arm and four in the observation arm. Grade 3 or 4 non-hematologic adverse events were observed in 25 patients $(28 \%)$ on lenalidomide and in none of the control group. The treatment discontinuation rate was $50 \%$, (in $40 \%$ of cases due to adverse events). Secondary primary malignancies occurred in $5.2 \%$ and $3.5 \%$ of treated and untreated patients, respectively. At the time of publication, data were not mature for evaluation of overall survival.

Several different, more intensive treatments are being or are close to being tested in SMM. Such approaches, briefly described below, generally comprise triplets including a proteasome inhibitor with an immunomodulatory drug + dexamethasone, monoclonal antibodies or even autologous stem cell transplantation. These new strategies, however, have so far generally been explored only in pilot studies with a limited number of patients or in phase II trials, with still preliminary results.

\section{Carfilzomib, lenalidomide and dexamethasone}

In a US pilot study (NCT01572480) in high-risk SMM, the carfilzomib, lenalidomide + dexamethasone (KRd) regimen resulted in an overall response rate of $100 \%{ }^{23,85}$ Very high rates of minimal-residual disease (MRD) negativity, as determined by multiparametric flow cytometry $(92 \%)$ or next-generation sequencing $(75 \%)$, were observed. After a median potential follow-up of 43.3 months, $63 \%$ of patients remained MRD-negative, with estimated 4-year progression-free and overall survival rates of $71 \%$ and $100 \%$, respectively. The safety profile was consistent with previous reports for this regimen.

A subsequent phase II study in high-risk SMM (Mayo Clinic or PETHEMA models) assessed KRd (8 cycles) + lenalidomide maintenance (KRd-R). ${ }^{86}$ After a median potential follow-up of 27.3 months, the overall response rate was $100 \%$ and $78 \%$ of patients achieved a best response of stringent complete remission. The primary objective of MRD-negative complete remission was achieved by $70.2 \%$, with a median duration of 5.5 years. At the 5 -year landmark, only $10 \%$ of patients had developed MM. No patient died, while grade 3-4 treatmentrelated adverse events occurred in $33 \%$ of patients, the most frequent being cytopenias, thromboembolism, rash, and lung infection.

Another ongoing multicenter, open-label phase II trial by the HOVON group is randomizing patients with high- risk SMM, defined according to both the Mayo Clinic ${ }^{52}$ and Spanish ${ }^{51}$ criteria, and with ultra-high-risk SMM, as defined by the IMWG, ${ }^{3}$ to treatment with a KRd combination versus lenalidomide + dexamethasone alone for nine cycles, followed by lenalidomide maintenance for 2 years (NCT03673826).

Among studies examining potentially curative strategies, the GEM-CESAR trial is a phase II, single-arm trial focusing on SMM patients at high risk of progression to active MM ( $>50 \%$ at 2 years), according to Spanish criteria (NCT02415413), and patients with ultra-high-risk SMM ${ }^{87,88}$ The induction therapy in this trial is six 4-week cycles of KRd, high-dose melphalan and autologous stem cell transplantation as intensification therapy, followed by two KRd consolidation cycles and maintenance with lenalidomide + dexamethasone for up to 2 years. The primary endpoint is a sustained MRD-negativity rate of at least $50 \%$, as determined by next-generation flow cytometry after induction and transplantation. At a first analysis of 90 patients younger than 70 years ${ }^{87}$ main grade 3-4 toxicities during induction and maintenance treatments included neutropenia (6\%), thrombocytopenia (11\%), infections $(18 \%)$ and skin rash $(9 \%)$. With a median follow-up of 32 months, $98 \%$ of patients remained alive and $93 \%$ progression-free, with only five patients having had biochemical relapses. Updated results ${ }^{88}$ indicate that the overall response rate was $98 \%$ after induction, $98 \%$ after autologous stem cell transplantation, and $100 \%$ after consolidation; $68.6 \%$ of patients reached complete remission or better after consolidation, with $55 \%$ of them achieving MRD negativity.

\section{Ixazomib-based regimens}

A combination of ixazomib + dexamethasone has been evaluated in a small pilot study in high-risk $\mathrm{SMM}^{89}$ (NCT02697383). At the time of the first analysis, with a median follow-up of 17 months, ten patients were continuing treatment: nine of them had achieved at least very good partial remission and no patient had progressed to symptomatic MM. Grade 3 non-hematologic adverse events included one intestinal complication and two grade 3 lung infections.

The oral triplet ixazomib, lenalidomide + dexamethasone for nine cycles followed by 15 cycles of ixazomib + lenalidomide was investigated in patients with high-risk SMM in a phase II trial..$^{\circ}$ Based on preliminary results in the first 26 patients, grade 3 hypophosphatemia, leukopenia, and neutropenia occurred in about $10 \%$ of patients, while $8 \%$ developed grade 4 neutropenia and hyperglycemia. The overall response rate in patients who received at least three cycles of treatment was 89\% (23/26), including five complete responses (19.2\%). None of the patients had shown progression to overt $\mathrm{MM}$ at the time of the analysis.

\section{Monoclonal antibodies}

As for other hematological malignancies, monoclonal antibodies are currently also being tested in SMM. ${ }^{91}$

\section{Daratumumab (anti-CD38)}

The randomized phase II CENTAURUS study (NCT02316106) evaluated daratumumab as a single agent in three different treatment schedules (extended intense, 
extended intermediate, or short dosing) in 123 patients with intermediate/high-risk SMM. ${ }^{92}$ The main endpoints were a complete remission rate $>15 \%$ and the progressive disease/death rate per patient-year ratio. After a median follow-up of 26 months, the complete remission rates were $4.9 \%, 9.8 \%$, and $0 \%$ for patients treated with the intense, intermediate, and short dosing schedules, respectively. Favorable progressive disease/death rate ratios and a median progression-free survival longer than 2 years were observed in all arms. Two-year progressionfree survival rates for intense, intermediate, and short dosing were $89.9 \%, 82 \%$, and $75.3 \%$, respectively. Target-saturating trough concentrations were generally preserved by intense dosing. No new safety issues occurred.

Based on these data, the long dosing schedule is being investigated in the ongoing, randomized phase III AQUILA study (NCT03301220) comparing subcutaneous daratumumab for up to 39 cycles versus active observation. ${ }^{93}$ The study will randomize about 360 patients with high-risk SMM according to one or more of the following features: serum $M$-component $\geq 3 \mathrm{~g} / \mathrm{dL}$, IgA SMM, immunoparesis, serum FLC ratio of $\geq 8$ but $<100$, BMPC percentage in the range of $51 \%$ to $60 \%$.

Another phase III, randomized trial is comparing lenalidomide + dexamethasone versus daratumumab, lenalidomide + dexamethasone in high-risk SMM (NCT03937635, DETER-SMM) patients, with overall survival as the primary endpoint. A target of 288 patients diagnosed within 1 year and having an abnormal serum FLC ratio $(\leq 0.125$ or $\geq 8.0)$ and involved chain $<100$ $\mathrm{mg} / \mathrm{L}$ ), and/or serum $\mathrm{M}$-component $>3 \mathrm{~g} / \mathrm{dL}$ and/or presence of $t(4 ; 14)$ or del $17 \mathrm{p}$ or $1 \mathrm{q}$ will be enrolled.

The ongoing, phase II ASCENT trial (NCT03289299) is evaluating daratumumab, carfilzomib, lenalidomide and dexamethasone (DKRd) without autologous stem cell transplantation in high-risk SMM. ${ }^{94}$ Treatment consists of 12 DKRd cycles, followed by maintenance with lenalidomide and daratumumab for 12 cycles. Preliminary safety data on 46 patients who reported grade 3-4 adverse events documented cytopenia, thromboembolic events, infections, hypertension, diarrhea, and allergic reactions, which all occurred in less than $10 \%$ of subjects. The relative median dose intensity was $\geq 80 \%$ for all drugs, demonstrating the feasibility of such an approach.

Single-agent daratumumab given intravenously for up to 20 cycles is also being evaluated in a phase II study in lower-risk SMM (NCT03236428). ${ }^{95}$ Feasibility was demonstrated in 28 patients. Response rates were assessed in 15 patients who completed six or more cycles: Partial response and at least very good partial response were achieved in $53 \%$ and $20 \%$ of these patients, respectively. Thus far, no deaths, progression or therapy discontinuations due to toxicity have occurred.

\section{Isatuximab (anti-CD38)}

A phase II study (NCT02960555) is exploring the efficacy of isatuximab $(20 \mathrm{mg} / \mathrm{kg}$ intravenously) in high-risk SMM; the drug is administered at decreasing intervals up to 30 weeks. $^{96}$ The median number of cycles given in the first 24 evaluable patients was 11.5 (range, 6-30). Five patients interrupted treatment, two because of progression to active MM. No deaths occurred during the study. Five grade 3 treatment-related adverse events [dyspnea, infusion related reactions, headache, neutropenia, urinary tract infections] were observed, all of which resolved to baseline. Best responses included partial remission (42\%), very good partial remission (17\%), and complete remission with MRD negativity by multiparameter flow cytometry at $10^{-5}(5 \%)$. This study also evaluated healthrelated quality of life scores, suggesting that isatuximab may reduce anxiety and worry of progression to $M M$, with improvement of the score after six cycles.

A phase III, randomized, multicenter study comparing isatuximab, lenalidomide + dexamethasone versus lenalidomide + dexamethasone in higher-risk SMM (according to IMWG criteria) within 5 years is about to start (NCT04270409). An initial safety run-in phase will confirm the recommended dose of isatuximab. Primary endpoints will include safety and efficacy (progressionfree survival), while pharmacokinetic and immunological studies, percentages, duration and quality of responses (including MRD), type of progression, second progression-free survival, overall survival, economic and healthrelated quality of life evaluations will be secondary objectives.

\section{Elotuzumab (anti-SLAMF7/CS1)}

Elotuzumab monotherapy had modest activity in SMM patients (overall response rate, $10 \%$,) in a phase II trial (NCT01441973). ${ }^{97}$ However, duration of response and the 2 -year progression-free survival of $69 \%$ in some patients with high-risk characteristics were of some interest. In that study, no relationship emerged between baseline $\mathrm{CD}^{6}{ }^{\mathrm{dim}} \mathrm{NK}$ cells and response.

Another phase II trial (NCT02279394), tested elotuzumab, lenalidomide + dexamethasone in high-risk SMM. ${ }^{98}$ This combination induced partial remissions or better, including three complete remissions and 18 very good partial remissions, in 41 of 50 patients (84\%). Genomic studies revealed that mutations occurring in genes involved in DNA repair were associated with poor response. No progression to symptomatic MM was observed and the toxicity profile was manageable, although some thromboembolic events occurred.

\section{Pembrolizumab (anti-PD1)}

The checkpoint inhibitor pembrolizumab was investigated in 13 patients with intermediate/high-risk SMM (NCT02603887). ${ }^{99}$ After a median of eight cycles, 11 patients $(85 \%)$ had stable disease, one patient progressed and one patient with $17 \mathrm{p}$ deletion and a high-risk geneexpression signature reached MRD negativity at $10^{-4}$ by next-generation sequencing in bone marrow which persisted after 27 months. Three patients discontinued the treatment due to immune-related adverse events.

\section{Siltuximab (anti-interleukin-6)}

A randomized, double-blind, placebo-controlled study evaluated interleukin- 6 blocking with siltuximab in 85 patients with high-risk SMM (NCT01484275). ${ }^{79}$ After a median follow-up of 29.2 months, the 1-year progression-free survival rate was $84.5 \%$ with siltuximab and $74.4 \%$ with placebo. The median progression-free survival was not reached with siltuximab whereas it was 23.5 months with placebo $(P=0.057)$. Adverse events in the experimental arm were mainly of grade $2-3$, the most common and serious ones being infections and urinary complications. Three deaths occurred with siltuximab and four with placebo. 


\section{Vaccines}

PVX-410 is a vaccine composed of a combination of four peptides, specifically targeting the highly overexpressed plasma cell antigens XBP1, CD138 and CS1/SLAMF7. A phase I/IIa multicenter, dose-escalation study (NCT01718899) accrued 22 patients with SMM at moderate or high risk of progression who received PVX410 , with or without lenalidomide ${ }^{100}$ The most common adverse events were mild-to-moderate injection site reactions and constitutional symptoms. PVX-410 was immunogenic as monotherapy and, more consistently, in combination with lenalidomide, as demonstrated by an increase in the percentage of PVX-410 tetramer and interferon- $\gamma$-positive specific $\mathrm{CD}^{+}{ }^{+} \mathrm{CD} 8^{+} \mathrm{T}$ lymphocytes, as well as by a persistent rise of vaccine-specific effector memory cells. In the PVX-410-alone cohort, three of 12 patients progressed, with a median time to progression of 36 weeks. In the combination cohort, five of 12 patients showed a clinical response, with one patient progressing and a median time to progression not reached. PVX-410 is also under investigation in SMM in combination with the selective histone-deacetylase inhibitor citarinostat \pm lenalidomide in a phase I trial (NCT02886065).

PD-L1 peptide vaccine, a new molecule targeting this immunological checkpoint, is also currently under investigation in SMM (NCT03850522).

\section{Other trials}

A phase II trial with ibrutinib (NCT02943473), a Bruton tyrosine kinase inhibitor, was recently closed due to poor accrual and an unfavorable risk/benefit ratio in patients with high-risk SMM.

\section{General considerations}

Despite the many ongoing and planned studies, only one randomized trial has so far demonstrated a significant survival benefit for early active therapy of high-risk $\mathrm{SMM}^{82}$ Another recent trial showed an improvement in progression-free survival, but follow-up is still too short for adequate evaluation of overall survival. ${ }^{84}$

Both these studies raised some concerns. Their sample size was limited, with less than 100 patients in each arm. The Spanish study was conducted between 2007 and $2013,{ }^{80}$ when some new MM drugs were not available, while bone involvement was assessed by a low-sensitivity technique such as plain radiography. Thus the survival benefit in some patients could have been due to treating active MM, rather than SMM. Furthermore, patients in the control group could start therapy only after meeting $C R A B$ criteria, which may be difficult in patients with progressive deterioration of renal function or anemia, because they would start therapy before the cut-off is reached. On the other hand, in the US trial, the high discontinuation rate and the fact that the group achieving the most significant benefit with lenalidomide in terms of progression-free survival included only 25 patients could be concerns. ${ }^{84}$

Notwithstanding, all ongoing studies seem to take a survival benefit for granted, as they do not include an untreated control group or compare two arms with active treatments, thus substantially accepting the philosophy that high-risk SMM "should" be treated. However, while some experts suggest that the Soutwest Oncology Group and Spanish trials should represent the current standard of care for patients with high-risk SMM, ${ }^{8,11}$ others are not convinced. ${ }^{10,14}$ Studies underway will likely show a response to therapy, more or less pronounced depending on the drugs used, with consequent improvement of progression-free survival. However, whether these often intensive treatments with novel agents will also have an extensive impact on overall survival of SMM patients will not be formally addressed and could remain unclear. The key issue remains to identify patients with high-risk SMM who "must" receive a treatment, because they will certainly have a significantly longer survival.

\section{Recommendations for clinical practice and future research}

\section{Which diagnostic procedures are necessary for diagnosing smoldering multiple myeloma?}

An adequate diagnostic work-up for SMM should include a hemogram and biochemistry with renal function and calcium levels, morphological and (if available) phenotypic quantification of clonal plasma cells in bone marrow smears and bone trephine biopsy, with cytogenetics by fluorescence in situ hybridization or a validated equivalent molecular method on purified plasma cells, evaluation of serum and urinary M-component, total serum immunoglobulins, serum involved/uninvolved FLC ratio and their absolute values. Diagnostic imaging should comprise low-dose whole-body CT and whole-body MRI, if low-dose whole-body CT is negative. Axial MRI or PETCT are reasonable alternatives, according to availability and specific diagnostic needs, as previously detailed.

\section{How should current predictive models be applied in the setting of smoldering multiple myeloma?}

Prediction of progression to overt MM of SMM should be based on routine and universally applicable tests, considering, however, that proposed models may show significant discordances in identifying "true" high-risk SMM. ${ }^{101}$ All the clinical prognostic scores reported above, particularly the updated 2/20/20 model (endorsed by the IMWG and including variables that reflect both disease burden and biological features of SMM that can be measured by most centers), represent important tools that physicians must routinely use (whatever they select) for risk stratification, to reasonably predict the outcome of different patients with SMM, because their management should be riskadapted. Combination with appropriate imaging investigation is also recommended.

Although more sophisticated genomic facilities may be available at specialized academic research institutions (where fluorescence in situ hybridization is replaced by tests such as whole exome sequencing), they are not mandatory, as not yet routinely used and validated for clinical purposes.

\section{Should patients with smoldering multiple myeloma be informed according to their different risk of evolution?}

Due to the heterogeneous behavior of SMM, appropriate information about their possible future clinical outcome should be given to patients with either lower- or 
higher-risk SMM, as defined by current risk models. The possibility of enrollment in a clinical trial, if available, should also be proposed.

\section{How should smoldering multiple myeloma be monitored?}

Once diagnosed, SMM patients should be monitored according to the above-reported recommendations, taking into particular account the individual risk of progression. Changes in serum $\mathrm{M}$-component represent the most simple, useful and worldwide available biomarker of progression over time. Serum FLC monitoring has also been demonstrated to be useful in this setting. ${ }^{102}$ However, for all SMM patients, it is important to follow the evolution of multiple different parameters that, taken together, may give better and comprehensive insight into the dynamics of the disease. Imaging should be periodically repeated, as previously detailed, and also performed if there is a biochemical or clinical suspicion of disease progression.

\section{Which patients with smoldering multiple myeloma might benefit from early treatment?}

Regarding patients with lower-risk SMM, diagnosed according to current criteria, only active observation is recommended.

With regard to early treatment of high-risk SMM, there is no consensus yet. Two prospective randomized trials have shown significant benefits from treatment with lenalidomide \pm dexamethasone in these patients, but they were not registration studies and they were not presented to regulatory agencies. ${ }^{80,84}$ However, it should be considered that patients presenting with the coexistence of multiple risk factors, particularly increasing $\mathrm{M}$-component levels or BMPC count or a significant decrease in hemoglobin concentration, high FLC ratio and/or high-risk cytogenetics, will further increase their risk of progression. For those cases, physicians may consider starting early treatment, with the intention of either delaying progression or even achieving a cure. However, it will be the individual physician's responsibility to seek active risk/benefit discussion with their patients, also considering that health-related quality of life is an essential outcome parameter. ${ }^{103}$ The decision regarding treatment will also depend on whether such an unlicensed treatment approach falls within the legal framework of the national healthcare system. The Expert Panel agreed that therapy in these selected, very high-risk SMM patients, should be similar to that offered to patients with active MM, and that their treatment should be administered in a controlled setting, such as a clinical trial. ${ }^{104}$

\section{What should be done in the near future to further improve the management of smoldering multiple myeloma?}

It is quite difficult to compare the results of the treatments that are currently being assessed in SMM, because of the substantial differences in times at which the studies were conducted, definitions of high-risk SMM, intensities of therapeutic approaches, and criteria/methods to evaluate response and progression across these studies. Thus, before definitively changing the current paradigms for the management of SMM, comparable future trials will have to be performed, aiming to define the following, relevant primary objectives: (i) to identify new predictive biomarkers (clinical, molecular/genomics, immunological, microenvironmental, imaging) for further refining risk prediction and selecting SMM patients who may do well with observation ("Dr. Jekyll") and those who require more stringent monitoring in order to establish the most appropriate moment to start treatment ("Mr. Hyde"). In this setting, possible racial diversities should also be considered, as they may have an impact on SMM biology; ${ }^{105}$ (ii) to assess the necessary balance between reduced risk of progression (and of consequent $\mathrm{MM}$ complications) with early treatment versus possible short- and long-term adverse effects, specifically deteriorating health-related quality of life, secondary primary malignancies and induction of refractory disease, elucidating, in particular, whether early treatment may select resistant clones or, the opposite, if delaying therapy may favor disease that is more resistant to future therapies; and (iii) to determine what intensity and which type of treatment are preferable in selected patients with high-risk SMM, i.e., short-term, intensive approaches with "curative" intent versus prolonged immunological control of the disease, for example by targeting immunity with memory to provide long-term surveillance, according to a "preventive" strategy. Both these approaches should have the primary objective of improving overall survival, without negatively affecting health-related quality of life.

\section{Disclosures}

PM has served as a member of advisory boards and/or received honoraria from Celgene, Janssen, Takeda, BristolMyers Squibb, Amgen, Novartis, Gilead, Jazz, Sanofi, Abbvie, and Glaxo-Smith-Kline. NB has received honoraria from Celgene, Takeda, Janssen, and Amgen; and served on an advisory board for Janssen. $M K$ has received consultancy fees from AbbVie, Amgen, BMS/Celgene, GSK, Janssen, Karyopharm, Seattle Genetics, and Takeda; honoraria from BMS/Celgene, Janssen, and Takeda; research funding from Janssen (to his institution), BMS/Celgene (to his institution); and travel support from BMS/Celgene, Janssen, and Takeda. NVDD has received research support from Janssen Pharmaceuticals, Amgen, Celgene, Novartis, and BMS; and served on advisory boards for Janssen Pharmaceuticals, Amgen, Celgene, BMS, Takeda, Roche, Novartis, Bayer, and Servier. ET has received consultancy fees and honoraria from Amgen, BMS, Janssen, Celgene, Takeda, Genesis Pharma, GSK and Sanofi; and research support from Amgen, Janssen, Celgene, Genesis Pharma, GSK and Sanofi. FG has received honoraria from Amgen, Celgene, Janssen, Takeda, BMS, AbbVie, and GSK; and has served on advisory boards for Amgen, Celgene, Janssen, Takeda, BMS, AbbVie, GSK, Roche, Adaptive Biotechnologies, and Oncopeptides. HG has received grants and/or provision of investigational medicinal products from Amgen, BMS, Celgene, Chugai, Dietmar-Hopp-Foundation, Janssen, John Hopkins University, Sanofi; research support (institutions) from Amgen, BMS, Celgene, Chugai, Janssen, Incyte, Molecular Partners, Merck Sharp and Dohme (MSD), Sanofi, Mundipharma, Takeda, and Novartis; served on advisory boards for Adaptive Biotechnology, Amgen, BMS, Celgene, Janssen, Sanofi, and Takeda; and honoraria (for speakers bureaus) from Academy2 GmbH \& Co. KG, Agentur Hogg Robinson Germany, Amgen, ArtTempi, Beupdated Helbig Consulting and Research AG Schweiz, BMS, Celgene, Chop GmbH, Chugai, Congress Culture Concept Dr. S. Stocker München, Connectmedia Warschau/Polen, Dr. Hubmann Tumorzentrum München, FomF GmbH, GlaxoSmithKline (GSK), GWT Forschung und Innovation Dresden, Institut für Versorgungsforschung in der 
Onkologie GbR, Janssen, Kompetenznetz Maligne Lymphome (KML) e.V., MedConcept GmbH, Medical Communication Gmbh, Münchner Leukämie Labor Prof. Haferlach, New Concept Oncology, Novartis, Omnia Med Deutschland, Onko Internetportal dkg-web GmbH, Sanofi, STIL Forschungs GmbH, and Veranstaltungskonzept Gesundheit Mechernich. RH has received research funding from Janssen, Amgen, Celgene, BMS, Novartis and Takeda; served on advisory boards for Janssen, Amgen, Celgene, AbbVie, BMS, Novartis, PharmaMar and Takeda; and has received honoraria from Janssen, Amgen, Celgene, BMS, Pharma Mar and Takeda. EZ has received honoraria from and served on advisory boards for Janssen, BMS, Takeda, Sanofi, Oncopeptide, GSK, and Amgen. SZ has received research funding from Takeda and Janssen; and served on advisory boards for Celgene, Takeda, Janssen, Sanofi and Oncopeptides. $M C$ has received honoraria from Janssen, Celgene, Amgen, BMS, Takeda, AbbVie, Sanofi, and Adaptive Biotechnologies, and is a member of speakers' bureaus for Janssen and Celgene. MD has received consultancy fees and honoraria from Janssen, Celgene, Takeda, Amgen and BMS. HL has received research funding from Amgen, Takeda; and served on speaker's bureauladvisory boards for Amgen, Takeda, Sanofi, Celgene-BMS, Seattle Genetics, and Janssen. MB has received honoraria from Sanofi, Celgene, Amgen, Janssen, Novartis, $B M S$, and AbbVie; served on advisory boards for Janssen and GSK; and has received research funding from Sanofi, Celgene, Amgen, Janssen, Novartis, BMS, and Mundipharma. M-VM has served on advisory boards for or received honoraria from Janssen, BMS, Celgene, Takeda, Amgen, Sanofi, Oncopeptides, GSK, Adaptive, Pfizer, Regeneron, Roche and Sea-Gen. PS has received honoraria and research funding from Amgen, Celgene, Janssen, SkylineDx, and Takeda; JSM has received consultancy fees from Amgen, BMS, Celgene, Janssen, MSD, Novartis, GSK Takeda, Sanofi, and Roche.

\section{Contributions}

$P M$ reviewed the literature and wrote the first draft. All authors revised, provided comments and consensus according to the Delphi methodology, and approved the final draft before submission.

\section{References}

1. Kyle RA, Greipp PR. Smoldering multiple myeloma. N Engl J Med. 1980;302(24):13471349.

2. Kyle RA, Child JA, Anderson $\mathrm{K}$, et al Criteria for the classification of monoclonal gammopathies, multiple myeloma and related disorders: a report of the International Myeloma Working Group. Br J Haematol. 2003;121(5):749-757.

3. Rajkumar SV, Dimopoulos MA, Palumbo A, et al. International Myeloma Working Group updated criteria for the diagnosis of multiple myeloma. Lancet Oncol. 2014;15 (12):e538-e548.

4. Caers J, Fernández de Larrea C, Leleu X, et al. The changing landscape of smoldering multiple myeloma: a European perspective. Oncologist. 2016;21(3):333-342.

5. Ravindran A, Bartley AC, Holton SJ, et al. Prevalence, incidence and survival of smoldering multiple myeloma in the United States. Blood Cancer J 2016;6(10):e486.

6. Kyle RA, Remstein ED, Therneau TM, et al. Clinical course and prognosis of smoldering (asymptomatic) multiple myeloma. $\mathrm{N}$ Engl J Med. 2007;356(25):2582-2590.

7. Rajkumar SV, Landgren O, Mateos M-V. Smoldering multiple myeloma. Blood. 2015;125(20):3069-3075.

8. Lonial S, Dhodapkar MV, Rajkumar SV. Smoldering myeloma and the art of war. J Clin Oncol. 2020;38(21):2363-2365

9. Ahn IE, Mailankody S, Korde N, Landgren O. Dilemmas in treating smoldering multiple myeloma. J Clin Oncol. 2015;33(1):115-123.

10. Fonseca R, Gonzalez-Velez M. Treatment of smoldering multiple myeloma: expectant observation should still be the standard. Am Soc Clin Oncol Educ Book. 2020;40:1-7.

11. Joseph NS, Dhodapkar MV, Lonial S. The role of early intervention in high-risk smoldering myeloma. Am Soc Clin Oncol Educ Book. 2020;40:1-9.

12. Biran N, Vesole DH, Donato ML, et al. Treatment of smoldering myeloma: problems with study design as well as biological and clinical implications. J Clin Oncol. 2020;38(12):1367-1368

13. Ho M, Patel A, Goh CY, Moscvin M, Zhang $\mathrm{L}$, Bianchi G. Changing paradigms in diag- nosis and treatment of monoclonal gammopathy of undetermined significance (MGUS) and smoldering multiple myeloma (SMM) Leukemia. 2020;34(12):3111-3125.

14. Goodman AM, Kim MS, Prasad V. Persistent challenges with treating multiple myeloma early. Blood. 2021;137(4):456-458

15. Bolli N, Sgherza N, Curci P, et al. What Is new in the treatment of smoldering multiple myeloma? J Clin Med. 2021;10(3):421.

16. Landgren O, Kyle RA, Pfeiffer RM, et al. Monoclonal gammopathy of undetermined significance (MGUS) consistently precedes multiple myeloma: a prospective study. Blood. 2009;113(22):5412-5417.

17. Morgan GJ, Walker BA, Davies FE. The genetic architecture of multiple myeloma. Nat Rev Cancer. 2012;12(5):335-348.

18. Boyle EM, Deshpande S, Tytarenko R, et al. The molecular make up of smoldering myeloma highlights the evolutionary pathways leading to multiple myeloma. Nat Commun. 2021;12(1):293.

19. Neben K, Jauch A, Hielscher T, et al. Progression in smoldering myeloma is independently determined by the chromosomal abnormalities del(17p), $t(4 ; 14)$, gain $1 q$, hyperdiploidy, and tumor load. J Clin Oncol. 2013;31(34):4325-4332

20. Zandecki M, Laï JL, Geneviève F, et al. Several cytogenetic subclones may be identified within plasma cells from patients with monoclonal gammopathy of undetermined significance, both at diagnosis and during the indolent course of this condition. Blood. 1997;90(9):3682-3690.

21. Drach J, Angerler J, Schuster J, et al. Interphase fluorescence in situ hybridization identifies chromosomal abnormalities in plasma cells from patients with monoclonal gammopathy of undetermined significance. Blood. 1995:86(10):3915-3921.

22. Oliva S, De Paoli L, Ruggeri M, et al. A longitudinal analysis of chromosomal abnormalities in disease progression from MGUS/SMM to newly diagnosed and relapsed multiple myeloma. Ann Hematol. 2021;100(2):437-443.

23. Mailankody S, Kazandjian D, Korde N, et al. Baseline mutational patterns and sustained MRD negativity in patients with high-risk smoldering myeloma. Blood Adv.
2017;1(22):1911-1918

24. Mikulasova A, Wardell CP, Murison A, et al. The spectrum of somatic mutations in monoclonal gammopathy of undetermined significance indicates a less complex genomic landscape than that in multiple myeloma. Haematologica. 2017;102(9):1617-1625.

25. Bolli N, Maura F, Minvielle S, et al. Genomic patterns of progression in smoldering multiple myeloma. Nat Commun. 2018;9(1): 3363 .

26. Ledergor G, Weiner A, Zada M, et al. Single cell dissection of plasma cell heterogeneity in symptomatic and asymptomatic myeloma. Nat Med. 2018;24(12):1867-1876.

27. Walker BA, Wardell CP, Melchor L, et al. Intraclonal heterogeneity is a critical early event in the development of myeloma and precedes the development of clinical symptoms. Leukemia. 2014;28(2):384-390.

28. Zhao S, Choi M, Heuck C, et al. Serial exome analysis of disease progression in premalignant gammopathies. Leukemia. 2014;28(7):1548-1552.

29. Dutta AK, Fink JL, Grady JP, et al. Subclonal evolution in disease progression from MGUS/SMM to multiple myeloma is characterised by clonal stability. Leukemia. 2019;33(2):457-468

30. Da Vià MC, Ziccheddu B, Maeda A, Bagnoli F, Perrone G, Bolli N. A journey through myeloma evolution: from the normal plasma cell to disease complexity. Hemasphere. 2020;4(6):e502.

31. Misund K, Keane N, Stein CK, et al. MYC dysregulation in the progression of multiple myeloma. Leukemia. 2020;34(1):322-326.

32. Oben B, Froyen G, Maclachlan KH, et al. Whole-genome sequencing reveals progressive versus stable myeloma precursor conditions as two distinct entities Nat Commun 2021 Mar 25;12(1):1861.

33. Maura F, Degasperi A, Nadeu F, et al. A practical guide for mutational signature analysis in hematological malignancies. Nat Commun. 2019;10(1):2969.

34. Maura F, Petljak M, Lionetti $M$, et al. Biological and prognostic impact of APOBEC-induced mutations in the spectrum of plasma cell dyscrasias and multiple myeloma cell lines. Leukemia. 2018;32(4): 1044-1048. 
35. Shah V, Johnson DC, Sherborne AL, et al. Subclonal TP53 copy number is associated with prognosis in multiple myeloma. Blood. 2018;132(23):2465-2469.

36. Manzoni M, Marchica V, Storti P, et al. Application of next-generation sequencing for the genomic characterization of patients with smoldering myeloma. Cancers (Basel). 2020;12(5):1332.

37. Manier S, Sacco A, Leleu X, Ghobrial IM, Roccaro AM. Bone marrow microenvironment in multiple myeloma progression. I Biomed Biotechnol. 2012;2012:157496.

38. Bianchi G, Munshi NC. Pathogenesis beyond the cancer clone(s) in multiple myeloma. Blood. 2015;125(20):3049-3058.

39. Nakamura K, Smyth MJ, Martinet L. Cancer immunoediting and immune dysregulation in multiple myeloma. Blood. 2020;136(24): 2731-2740.

40. Das R, Strowig T, Verma R, et al. Microenvironment-dependent growth of preneoplastic and malignant plasma cells in humanized mice. Nat Med. 2016;22(11): 1351-1357.

41. Zavidij O, Haradhvala NJ, Mouhieddine TH, et al. Single-cell RNA sequencing reveals compromised immune microenvironment in precursor stages of multiple myeloma. Nat Cancer. 2020;1(5):493-506

42. Wu V, Moshier E, Leng S, et al. Risk stratification of smoldering multiple myeloma: predictive value of free light chains and group-based trajectory modeling. Blood Adv. 2018;2(12):1470-1479.

43. Hillengass J, Usmani S, Rajkumar SV, et al. International myeloma working group consensus recommendations on imaging in monoclonal plasma cell disorders. Lancet Oncol. 2019:20(6):e302-e312.

44. Jamet B, Bailly C, Carlier T, et al. Imaging of monoclonal gammapathy of undetermined significance and smoldering multiple myeloma. Cancers (Basel). 2020;12(2):486.

45. Hillengass J, Moulopoulos LA, Delorme S, et al. Whole-body computed tomography versus conventional skeletal survey in patients with multiple myeloma: a study of the International Myeloma Working Group. Blood Cancer J. 2017;7(8):e599.

46. Gavriatopoulou M, Boultadaki A, Koutoulidis V, et al. The role of low dose whole body CT in the detection of progression of patients with smoldering multiple myeloma. Blood Cancer J. 2020;10(9):93,

47. Wennmann M, Hielscher T, Kintzelé L, et al. Spatial distribution of focal lesions in wholebody MRI and influence of MRI protocol on staging in patients with smoldering multiple myeloma according to the new SLiM-CRAB criteria. Cancers (Basel). 2020;12(9):2537.

48. Cavo M, Terpos E, Nanni C, et al. Role of 18F-FDG PET/CT in the diagnosis and management of multiple myeloma and other plasma cell disorders: a consensus statement by the International Myeloma Working Group. Lancet Oncol. 2017:18(4):e206-e217.

49. Kyle RA, Durie BGM, Rajkumar SV, et al. Monoclonal gammopathy of undetermined significance (MGUS) and smoldering (asymptomatic) multiple myeloma: IMWG consensus perspectives risk factors for progression and guidelines for monitoring and management. Leukemia. 2010;24(6):11211127

50. Cocito F, Mangiacavalli S, Ferretti VV, et al. Smoldering multiple myeloma: the role of different scoring systems in identifying high-risk patients in real-life practice. Leuk Lymphoma. 2019;60(12):2968-2974.

51. Pérez-Persona E, Vidriales M-B, Mateo G, et al. New criteria to identify risk of progres- sion in monoclonal gammopathy of uncertain significance and smoldering multiple myeloma based on multiparameter flow cytometry analysis of bone marrow plasma cells. Blood. 2007;110(7):2586-2592

52. Dispenzieri A, Kyle RA, Katzmann JA, et al. Immunoglobulin free light chain ratio is an independent risk factor for progression of smoldering (asymptomatic) multiple myeloma. Blood. 2008:111(2):785-789.

53. Waxman AJ, Mick R, Garfall AL, et al Classifying ultra-high risk smoldering myeloma. Leukemia. 2015;29(3):751-753

54. Sørrig R, Klausen TW, Salomo M, et al. Smoldering multiple myeloma risk factors for progression: a Danish population-based cohort study. Eur J Haematol. 2016;97 (3):303-309

55. Fernández de Larrea C, Isola I, Pereira A, et al. Evolving M-protein pattern in patients with smoldering multiple myeloma: impact on early progression. Leukemia. 2018;32(6): 1427-1434

56. González-Calle V, Dávila J, Escalante F, et al. Bence Jones proteinuria in smoldering multiple myeloma as a predictor marker of progression to symptomatic multiple myeloma. Leukemia. 2016;30(10):2026-2031

57. Ravi P, Kumar S, Larsen JT, et al. Evolving changes in disease biomarkers and risk of early progression in smoldering multiple myeloma. Blood Cancer J. 2016;6(7):e454.

58. Bustoros M, Kastritis E Sklavenitis-Pistofidis R, et al. Bone marrow biopsy in low-risk monoclonal gammopathy of undetermined significance reveals a novel smoldering multiple myeloma risk group. Am J Hematol. 2019;94(5):E146E149.

59. Sanoja-Flores L, Flores-Montero J, Garcés JJ, et al. Next generation flow for minimally invasive blood characterization of MGUS and multiple myeloma at diagnosis based on circulating tumor plasma cells (CTPC). Blood Cancer J. 2018;8(12):117

60. Aljama MA, Sidiqi MH, Lakshman A, et al. Plasma cell proliferative index is an independent predictor of progression in smoldering multiple myeloma. Blood Adv. 2018; (22):3149-3154

61. Merz M, Hielscher T, Wagner B, et al. Predictive value of longitudinal whole-body magnetic resonance imaging in patients with smoldering multiple myeloma. eukemia. 2014:28(9):1902-1908.

62.Zamagni E, Nanni C, Gay F, et al. 18F-FDC PET/CT focal, but not osteolytic, lesion predict the progression of smoldering myeloma to active disease. Leukemia. 2016;30(2):417-422.

63. Rajkumar SV, Gupta V, Fonseca R, et al. Impact of primary molecular cytogenetic abnormalities and risk of progression in moldering multiple myeloma. Leukemia. 2013;27(8):1738-1744

64. Khan R, Dhodapkar M, Rosenthal A, et al Four genes predict high risk of progression from smoldering to symptomatic multiple myeloma (SWOG S0120). Haematologica. 2015;100(9):1214-1221.

65. Merz M, Hielscher T, Schult D, et al Cytogenetic subclone formation and evolution in progressive smoldering multiple myeloma. Leukemia. 2020;34(4):1192-1196

66. Visram A, Soof C, Rajkumar SV, et al. Serum BCMA levels predict outcomes in MGUS and smoldering myeloma patients. Blood Cancer J. 2021;11(6):120

67. Dhodapkar MV, Sexton R, Waheed S, et al Clinical, genomic, and imaging predictors of myeloma progression from asymptomatic monoclonal gammopathies (swog s0120).
Blood. 2014:123(1):78-85.

68. Lakshman A, Rajkumar SV, Buadi FK, et al Risk stratification of smoldering multiple myeloma incorporating revised IMWG diagnostic criteria. Blood Cancer J. 2018;8(6):59.

69. Mateos MV, Kumar S, Dimopoulos MA, et al. International Myeloma Working Group risk stratification model for smoldering multiple myeloma (SMM). Blood Cancer J. 2020;10(10):102

70. Hájek R, Sandecka V, Špička I, et al Identification of patients with smouldering multiple myeloma at ultra-high risk of progression using serum parameters: the Czech Myeloma Group model. Br J Haematol. 2020;190(2):189-197.

71. Bustoros M, Sklavenitis-Pistofidis R, Park J, et al. Genomic profiling of smoldering multiple myeloma identifies patients at a high risk of disease progression. J Clin Oncol. 2020;38(21):2380-2389.

72. Zhao AL, Shen KN, Wang JN, Huo LQ, L J, Cao XX. Early or deferred treatment of smoldering multiple myeloma: a metaanalysis on randomized controlled studies. Cancer Manag Res. 2019;11:5599-5611

73. Hjorth M, Hellquist L, Holmberg E Magnusson B, Rödjer S, Westin J. Initial versus deferred melphalan-prednisone therapy for asymptomatic multiple myeloma stage a randomized study. Eur J Haematol. 2009;50(2):95-102

74. Riccardi A, Ucci G, Luoni R, et al. Treatment of multiple myeloma according to the extension of the disease: a prospective, randomised study comparing a less with a more aggressive cytostatic policy. $\mathrm{Br} \mathrm{J}$ Cancer. 1994;70(6):1203-1210.

75. Riccardi A, Mora O, Tinelli C, et al. Longterm survival of stage I multiple myeloma given chemotherapy just after diagnosis o at progression of the disease: a multicentre randomized study. Br J Cancer. 2000;82(7): 1254-1260.

76. Witzig TE, Laumann KM, Lacy MQ, et al. A phase III randomized trial of thalidomide plus zoledronic acid versus zoledronic acid alone in patients with asymptomatic multiple myeloma. Leukemia. 2013;27(1):220225

77. Musto P, Petrucci MT, Bringhen S, et al. A multicenter, randomized clinical trial comparing zoledronic acid versus observation in patients with asymptomatic myeloma. Cancer. 2008:113(7):1588-1595.

78. D'Arena G, Gobbi PG, Broglia C, et al. Pamidronate versus observation in asymptomatic myeloma: final results with long-term follow-up of a randomized study. Leuk Lymphoma. 2011;52(5):771-775.

79. Brighton TA, Khot A, Harrison SJ, et al Randomized, double-blind, placebo-controlled, multicenter study of siltuximab in high-risk smoldering multiple myeloma. Clin Cancer Res. 2019;25(13):3772-3775.

80. Mateos MV, Hernández MT, Giraldo P, et al Lenalidomide plus dexamethasone for highrisk smoldering multiple myeloma. $\mathrm{N}$ Engl J Med. 2013;369(5):438-447.

81. Mateos MV, Hernández MT, Giraldo P, et al Lenalidomide plus dexamethasone versus observation in patients with high-risk smouldering multiple myeloma (QuiRedex) long-term follow-up of a randomised, controlled, phase 3 trial. Lancet Oncol. 2016;17(8):1127-1136.

82. Mateos M-V, Hernandez MT, Salvador C, et al. Over ten years of follow-up for phase I trial in smoldering myeloma at high risk of progression to myeloma: sustained TTP and OS benefit with RD versus no treatment. Hemasphere. 2020;294867:EP950. 
83. Paiva B, Mateos MV, Sanchez-Abarca LI, et al. Immune status of high-risk smoldering multiple myeloma patients and its therapeutic modulation under LenDex: a longitudinal analysis. Blood. 2016;127(9):1151-1162.

84. Lonial S, Jacobus S, Fonseca R, et al. Randomized trial of lenalidomide versus observation in smoldering multiple myeloma. J Clin Oncol. 2020;38(11):1126-1137.

85. Korde N, Roschewski M, Zingone A, et al. Treatment with carfilzomib-lenalidomidedexamethasone with lenalidomide extension in patients with smoldering or newly diagnosed multiple myeloma. JAMA Oncol. 2015;1(6):746-754.

86. Kazandjian D, Hil E, Morrison C, et al. Background: Treatment of high risk (HR) smoldering multiple myeloma (SMM) with carfilzomib, lenalidomide, and dexamethasone (KRd) followed by lenalidomide maintenance $(-R)$ : a phase 2 clinical and correlative study. Blood. 2020;136(1):43-45.

87. Mateos M-V, Martinez-Lopez J, Rodriguez Otero P, et al. Curative strategy (GEMCESAR) for high-risk smoldering myeloma (SMM): carfilzomib, lenalidomide and dexamethasone (KRd) as induction followed by HDT-ASCT, consolidation with $\mathrm{KRd}$ and maintenance with $\mathrm{Rd}$. Blood. 2019;134(1):781.

88. Puig N, Contreras T, Paiva B, et al. Analysis of treatment efficacy in the GEM-CESAR trial for high-risk smoldering multiple myeloma patients: comparison between the standard and IMWG MRD criteria and OIPMS including FLC (OIP-FLC-MS). J Clin Oncol. 2020;38(15):8512.

89. Mailankody S, Salcedo M, Tavitian E, et al. Ixazomib and dexamethasone in high risk smoldering multiple myeloma: a clinical and correlative pilot study. J Clin Oncol. 2019;37(15):8051.
90. Bustoros M, Liu C, Reyes K, et al. Phase II trial of the combination of ixazomib, lenalidomide, and dexamethasone in highrisk smoldering multiple myeloma. Blood. 2018;132(1):804.

91. Musto P, La Rocca F. Monoclonal antibodies in newly diagnosed and smoldering multiple myeloma: an updated review of current clinical evidence. Expert Rev Hematol. 2020;13(5):501-517

92. Landgren CO, Chari A, Cohen YC, et al Daratumumab monotherapy for patients with intermediate-risk or high-risk smoldering multiple myeloma: a randomized, openlabel, multicenter, phase 2 study (CENTAU RUS). Leukemia. 2020;34(7):1840-1852.

93. Rajkumar SV, Voorhees PM, Goldschmidt $\mathrm{H}$, et al. Randomized, open-label, phase 3 study of subcutaneous daratumumab (DARA SC) versus active monitoring in patients (Pts) with high-risk smoldering multiple myeloma (SMM): AQUILA. J Clin Oncol. 2018;36(15):TPS8062.

94. Kumar SK, Abdallah AO, Badros AZ, et al Aggressive smoldering curative approach evaluating novel therapies (ASCENT): a phase 2 trial of induction, consolidation and maintenance in subjects with high risk smoldering multiple myeloma (SMM): Initial analysis of safety data. Blood. 2020;136(1):35 36.

95. Nadeem O, Redd R, Stampleman LV, et al. A phase II study of daratumumab in patients with high-risk MGUS and low-risk smoldering multiple myeloma: first report of efficacy and safety. Blood. 2019;134(1):1898.

96. Manasanch EE, Jagannath S, Lee HC, et al. A multicenter phase II single arm trial of isatuximab in patients with high risk smoldering multiple myeloma (HRSMM). Blood. 2019;134(1):3116

97. Jagannath S, Laubach J, Wong E, et al.
Elotuzumab monotherapy in patients with smouldering multiple myeloma: a phase 2 study. Br J Haematol. 2018;182(4):495-503.

98. Liu C, Ghobrial IM, Bustoros M, et al. Phase II trial of combination of elotuzumab, lenalidomide, and dexamethasone in highrisk smoldering multiple myeloma. Blood. 2018;132(1):154.

99. Manasanch EE, Han G, Mathur R, et al. A pilot study of pembrolizumab in smoldering myeloma: report of the clinical, immune, and genomic analysis. Blood Adv 2019;3(15):2400-2408.

100. Nooka AK, Wang ML, Yee AJ, et al Assessment of safety and immunogenicity of PVX-410 vaccine with or without lenalidomide in patients with smoldering multiple myeloma: a nonrandomized clinical trial. JAMA Oncol. 2018;4(12):e183267.

101. Hill E, Dew A, Morrison C, et al Assessment of discordance among smoldering multiple myeloma risk models. JAMA Oncol. 2021;7(1):132-134.

102. Gran C, Luong V, Bruchfeld JB, et al Dynamic follow-up of smoldering multiple myeloma identifies a subset of patients at high risk of progression. Am J Hematol. 2021;96(3):E63-E65.

103. Jean-Baptiste M, Gries KS, Lenderking WR Fastenau J. Symptom burden and healthrelated quality of life impacts of smoldering multiple myeloma: the patient perspective. J Patient Rep Outcomes. 2020;4(1):95.

104.Dimopoulos MA, Moreau P, Terpos E, et al. Multiple myeloma: EHA-ESMO clinical practice guidelines for diagnosis, treatment and follow-up. Ann Oncol. 2021;32(3):309322.

105. Marinac CR, Ghobrial IM, Birmann BM Soiffer J, Rebbeck TR. Dissecting racial disparities in multiple myeloma. Blood Cancer J. 2020;10(2):19. 